\title{
Towards an understanding of variations in the buckling of tailored variable angle tow composite plates
}

\author{
Xiao-Yi Zhou ${ }^{\mathrm{a}, *}$, P. D. Gosling ${ }^{\mathrm{b}}$ \\ ${ }^{a}$ School of Engineering, Cardiff University, Cardiff, CF24 3AA, UK. \\ ${ }^{b}$ School of Engineering, Newcastle University, Newcastle upon Tyne, NE1 7RU, UK.
}

\begin{abstract}
In this paper, variable angle tow (VAT) composite plates tailored to enhance buckling performance are studied with the use of stochastic finite element method to quantify uncertainties in buckling measures arising from variations in material properties and fibre tow path. Detailed formulations for predicting buckling statistics in terms of mean value and standard deviation are derived to enable a perturbation-based stochastic finite element analysis. The derivations are built on a linear variation formula for fibre tow path and plate element based on the first order shear deformation theory. They are integrated with Taylor series expansion to propagate uncertainties from inputs to buckling performance measures, including buckling eigenvalues, critical buckling coefficients, etc. A twelve-layer VAT composite plate, with optimally designed fibre tow paths under various boundary conditions, has been investigated to illustrate the uncertainty quantification procedure. The performance of the perturbation-based stochastic finite element method has
\end{abstract}

\footnotetext{
*Corresponding author

Email addresses: zhoux19@cardiff.ac.uk (Xiao-Yi Zhou), p.d.gosling@newcastle.ac.uk (P. D. Gosling)
} 
been validated using Monte Carlo simulation. Influences of variations in material properties and fibre tow path are thoroughly examined to understand the variability of buckling statistics of VAT composites.

Keywords: Buckling tailoring; Variable angle tow (VAT); Laminated composite plates; Uncertainty quantification; Stochastic finite element method.

\section{1. Introduction}

The freedom to obtain tailored properties is one of the most attractive 3 features of fibre reinforced polymer composites for developing lightweight

structures, achieved using well established techniques, e.g. the specific selection of new constituent materials or different compositions, designing alternative material architectures, etc. Recently, more advanced composite manufacturing techniques have emerged, such as variable angle tow (VAT) or tow steering composites, in which the fibre path is continuously varied to tailor the local stiffness properties of laminates, further enhancing their structural performance. VAT composites are receiving significant attentions from several industry sectors such as aerospace, where it has shown great potential in structures with high demands on buckling, aeroelasticity, and vibration with restricted weight allowance. However, this additional design freedom is at the expense of complexity in maintaining uniform fibre paths during manufacturing and increased computational cost. One of the main additional computational costs comes from finding the optimal fibre tow path (Hyer and Charette, 1991; Setoodeh et al., 2009; Ijsselmuiden et al., 2010; Wu et al., 2012; Sabido et al., 2017). In addition, the nature of its curved 
fibre courses means that they are inevitably more vulnerable to manufacturing defects, which have been recognized as the main sources of variations in structural performances, compared with unidirectional (UD) or straight fibre composites. It is thus desirable to understand the uncertainties of this type of advanced composite to provide confidences in their applications.

VAT composites are relatively new, meaning that reports on quantifying uncertainties in either material properties or structural performance are seldom available. Variations in fibre tow path have, however, been reported in (Kim et al., 2014). Due to the hierarchical nature of composites, relatively complex manufacturing technologies are required, with the manufacturing processes becoming the primary source for introducing material and geometric uncertainties. As a type of product using Automated Fibre Placement (AFP) technologies, VAT composites may share many manufacturing defects and variations in their properties with conventional composites. In general, variability exists in the constituent material properties arising from defects, such as fibre misalignment, waviness, etc. (Potter et al., 2008). It has been widely accepted that defects and their inherent variations are some of the main sources of damage initiation (Hörrmann et al., 2016). The formations of defects were investigated in (Belnoue et al., 2017; Lukaszewicz et al., 2012). For instance, one of the processes known to influence the formation of fibre path defects is the consolidation of laminates manufactured by AFP. Moreover, these defects are random in nature. Uncertainty in the geometry of fibre preforms manufactured with Automated Dry Fibre Placement and its effects on permeability were studied in (Matveev et al., 2017). The influence of manufacturing uncertainties on mechanical and/or physical properties of 
conventional composites, e.g. UD or textile, has been continuously investigated through numerical and experimental approaches, e.g. (Endruweit et al., 2013; Bednarcyk et al., 2014; Zhou et al., 2017; Zhou and Gosling, 2018). In the present study, the influences on buckling performance are of interest.

Uncertainty-driven probabilistic buckling analysis has been historically researched for composite structures. Lin et al. (1998) investigated the buckling failure probability evaluation of laminated composite plates subjected to different in-plane random loads using the stochastic finite element method, with the feasibility and accuracy of the results validated using results obtained from Monte Carlo Simulations (MCS). Using alternative methods, reliability predictions of laminated composite plates with random system parameters subjected to transverse loads have also been made (Lin, 2000). Singh et al. (2001) studied the effects of random material properties on the buckling of composite plates. Orifici and Bisagni (2013) used a perturbationbased technique to investigate the impacts of imperfections on the buckling of composite cylindrical shells. Chen and Guedes Soares (2007) conducted a reliability analysis of post-buckling performance. In addition to mechanical load induced buckling, other actions, e.g. temperature-induced buckling has also been reported. Lal et al. (2009) investigated the effects of random system properties on the thermal buckling of composites. Lal et al. (2011) further conducted a stochastic post-buckling analysis of laminated composites subjected to hygro-thermal-mechanical loading. The effect of random system properties, plate geometry, stacking sequences, supporting conditions, and fibre volume fractions on hygro-thermal-mechanical buckling 
load of the laminated shells and panels were investigated using a first order perturbation-based stochastic finite element method (PSFE). Most recently, Li et al. (2016) conducted a stochastic thermal buckling analysis using a perturbation technique to propagate uncertainties from the constituent material scale to the structural scale. It is noted that the perturbation-based finite element method is the most commonly adopted technique for probabilistic buckling analysis. However, all the aforementioned studies have only investigated conventional unidirectional fibre reinforced polymer composites. No equivalent studies appear to have been published for VAT composites.

In this study, the perturbation-based stochastic finite element method is adopted to develop an uncertainty quantification approach to investigate the buckling characteristics of VAT composite plates subject to mechanical loads e.g. uniform end shortening displacement. The first-order shear deformation theory, which enables to consider transverse shear strain that can provide sufficiently accurate prediction of the structural response for the medium thickness laminated plates, is used as the basis to develop the stochastic finite element method. As the primary objective of the present study is to understand the variations in buckling performance, relatively simple VAT composite plates with a linear variation to define fibre tow path (see Figure 1b based on the formula proposed by Gürdal et al. (2008)) are considered to investigate the mechanical characteristics of such structures. However, the method described in this paper can be straightforwardly applied to VAT composites with more complicated fibre path functions, such as Lagrangian polynomials (see Figure 1c) proposed by Wu et al. (2012). In the present study, the formulation for propagating uncertainties from material properties 
e.g. moduli and Poisson's ratios and fibre tow path e.g. rotation angle in the linear variation formula to buckling performance measures, i.e. eigenvalue and critical buckling coefficients, is derived and given in the form of a finite element analysis. Statistics, namely mean values and standard deviations, of the buckling measures are given. The proposed uncertainty quantification procedure is demonstrated through studies on a VAT composite plate tailored for maximum buckling performance, under alternative boundary conditions, and subjected to uniform displacement shortening. Monte Carlo simulation is used to validate the performance of the method in terms of accuracy and efficiency. Further studies are conducted to investigate the stochastic mechanical behaviours of VAT composite plates in the presence of uncertainties in material properties and fibre tow path directions.

\section{Methodology}

\subsection{Fibre path formulation}

Whilst nonlinear tow angle descriptions have been shown to replacedprovide an extended design space be advantageous when endeavouring to optimize the performance of VAT composite plates, a linear tow angle variation proposed by Gürdal et al. (2008) is adopted here as the primary purpose of the current study is to quantify the uncertainties in buckling performance of VAT composite structures. The simpler description of fibre tow path can be easier to evaluate the variations in mechanical behaviour of VAT composites, while the stochastic analysis procedure developed in the following sections is straightforward to incorporate with other fibre path functions. The linear variation of fibre tow path adopted in the present study is illustrated in 
Figure 1a and expressed as:

$$
\theta(x)=\phi+T_{0}+\left(T_{1}-T_{0}\right) \frac{\left|x^{\prime}\right|}{d}
$$

where $T_{0}$ and $T_{1}$ are the angles at two control points, $A$ and $B$, respectively, $d$ is the distance between these two points, $\phi$ is the direction moving from $\mathrm{A}$ to $\mathrm{B}$, and $x^{\prime}=x \cos (\phi)+y \sin (\phi)$. With this formula, a single VAT composite layer may be represented by $\phi<T_{0} \mid T_{1}>$, and laminates with multiple VAT composite layers can be specified by different combinations of the angle $\phi$ and stacks of $\pm<T_{0} \mid T_{1}>$.

\subsection{Linear buckling analysis of variable angle tow laminated composite plates}

For the structural analysis of VAT laminated composite plates, one of the essential differences comparing with conventional composite laminates, is that the stiffness properties now vary as a function of spatial location. Therefore, in the finite element analysis context, the discretized domain will produce locally straight fibre paths; each element will have a local fibre angle associated with it (see Figure 2).

For a linear buckling analysis, the buckling load is determined through the following eigenvalue problem:

$$
\left(\mathbf{K}-\lambda \mathbf{K}_{g}\right) \mathbf{V}=\mathbf{0}
$$

where $\mathbf{V}=\left\{\boldsymbol{v}_{1}, \cdots, \boldsymbol{v}_{n}\right\}$ is the right-hand eigenvector corresponding to eigenvalues of $\boldsymbol{\lambda}=\left\{\lambda_{1}, \cdots, \lambda_{n}\right\}$ with $n$ for the total number of eigenmodes, $\mathbf{K}$ is the global stiffness matrix, and $\mathbf{K}_{g}$ is the global geometric stiffness matrix due to an applied mechanical load. 
In the present study, the above equation is solved using four-node quadrilateral plate elements based on the first order shear deformation theory. Each node has five degrees of freedom, including three translations $u, v, w$ and two rotations $\theta_{x}, \theta_{y}$. Briefly, to solve it, a prebuckling analysis is conducted first to find structural responses under a given loading condition to assemble $K_{g}$, and the equation is solved with an eigensolver. This is conducted with an in-house program developed in MATLAB. In what follows, we briefly present this computational scheme, following the notations adopted by (Oñate, 2013). The global stiffness matrix is thus written as:

$$
\mathbf{K}=\sum_{e=1}^{N E} \mathbf{k}_{e}=\sum_{e=1}^{N E}\left[\mathbf{B}^{T} \hat{\mathbf{D}}_{e} \mathbf{B}\right]=\sum_{e=1}^{N E} \sum_{k=1}^{N P}\left[\mathbf{B}^{T} \mathbf{L}_{e k}^{T} \mathbf{D}_{e k} \mathbf{L}_{e k} \mathbf{B}\right]
$$

where the superscript $T$ denotes a vector or matrix transposition; $\mathbf{k}_{e}$ is the element stiffness matrix with the dimension of $20 \times 20$; $\mathbf{B}$ is the straindisplacement transformation matrix with the dimension of $8 \times 20$ (see e.g. (Oñate, 2013)); $\hat{\mathbf{D}}_{e}$ is the generalized constitutive matrix with the dimension of $8 \times 8 ; N E$ and $N P$ are the numbers of elements and plies, respectively; $\mathbf{D}_{e k}$ is the constitutive matrix of the $k$-th ply for the $e$-th element given by:

$$
\underset{8 \times 8}{\mathbf{D}_{e k}}=\left[\begin{array}{ccc}
t_{k} \mathbf{C}_{1 k} & -t_{k} \bar{z}_{k} \mathbf{C}_{1 k} & \mathbf{0} \\
-t_{k} \bar{z}_{k} \mathbf{C}_{1 k} & \frac{1}{3}\left(z_{k+1}^{3}-z_{k}^{3}\right) \mathbf{C}_{1 k} & \mathbf{0} \\
\mathbf{0} & \mathbf{0} & \kappa \mathbf{C}_{2 k}
\end{array}\right]
$$

where $t_{k}=z_{k+1}-z_{k}$ is the thickness of $k$-th ply, $\bar{z}_{k}=\frac{1}{2}\left(z_{k}+z_{k+1}\right)$ is the midplane of the $k$-th ply, $z_{k+1}$ and $z_{k}$ are the through-thickness coordinate of the top and bottom surface of the $k$-th ply, and $\kappa$ is the shear correction factor, 5/6 used here; $\mathbf{C}_{1 k}$ and $\mathbf{C}_{2 k}$ are the in-plane constitutive and out-plane shear constitutive matrices of the $k$-th ply. For example, $\mathbf{C}_{1 k}$ and $\mathbf{C}_{2 k}$ for a lamina 
made of a transversely isotropic material, such as the carbon fibre reinforced polymer composite investigated the following numerical study section, are:

$$
\left.\underset{3 \times 3}{\mathbf{C}_{1 k}}=\left[\begin{array}{ccc}
\frac{E_{1 k}^{2}}{E_{1 k}-E_{2 k} \nu_{12 k}^{2}} & \frac{E_{1 k} E_{2 k} \nu_{12 k}}{E_{1 k}-E_{2 k} \nu_{12 k}^{2}} & 0 \\
\frac{E_{1 k} E_{2 k} \nu_{12 k}}{E_{1 k}-E_{2 k} \nu_{12 k}^{2}} & \frac{E_{1 k} E_{2 k}}{E_{1 k}-E_{2 k} \nu_{12 k}^{2}} & 0 \\
0 & 0 & G_{12 k}
\end{array}\right], \quad \begin{array}{c}
\mathbf{C}_{2 \times 2} \\
0
\end{array}\right]=\left[\begin{array}{cc}
G_{23 k} & 0 \\
0 & G_{13 k}
\end{array}\right]
$$

$$
\begin{aligned}
& \underset{3 \times 3}{\mathbf{T}_{1 e k}}= {\left[\begin{array}{ccc}
\cos ^{2} \theta_{e k} & \sin ^{2} \theta_{e k} & \sin \theta_{e k} \cos \theta_{e k} \\
\sin ^{2} \theta_{e k} & \cos ^{2} \theta_{e k} & -\sin \theta_{e k} \cos \theta_{e k} \\
-2 \sin \theta_{e k} \cos \theta_{e k} & 2 \sin \theta_{e k} \cos \theta_{e k} & \cos ^{2} \theta_{e k}-\sin ^{2} \theta_{e k}
\end{array}\right], } \\
& \underset{\substack{2 \times 2 \\
\mathbf{T}_{2 e k}}}{ }=\left[\begin{array}{cc}
\cos \theta_{e k} & -\sin \theta_{e k} \\
-\sin \theta_{e k} & \cos \theta_{e k}
\end{array}\right]
\end{aligned}
$$

with

$$
\underset{8 \times 8}{\mathbf{L}_{e k}}=\left[\begin{array}{ccc}
\mathbf{T}_{1 e k} & \mathbf{0} & \mathbf{0} \\
\mathbf{0} & \mathbf{T}_{1 e k} & \mathbf{0} \\
\mathbf{0} & \mathbf{0} & \mathbf{T}_{2 e k}
\end{array}\right]
$$

here $\theta_{e k}$ is the corresponding fibre tow orientation (see Figure 2).

The geometric stiffness matrix $\mathbf{K}_{g}$ consists of contributions from both bending, $\mathbf{K}_{g b}$, and shear, $\mathbf{K}_{g s}$, and is written as (Ferreira, 2009):

$$
\mathbf{K}_{g}=\mathbf{K}_{g b}+\mathbf{K}_{g s}
$$


with

$$
\begin{aligned}
\mathbf{K}_{g b} & =\sum_{e=1}^{N E}\left(\mathbf{B}_{g b 1}^{T} \mathbf{S}_{e} \mathbf{B}_{g b 1}+\mathbf{B}_{g b 2}^{T} \mathbf{S}_{e} \mathbf{B}_{g b 2}+\mathbf{B}_{g b 3}^{T} \mathbf{S}_{e} \mathbf{B}_{g b 3}\right) \\
\mathbf{K}_{g s} & =\sum_{e=1}^{N E}\left(\mathbf{B}_{g s 1}^{T} \mathbf{S}_{e} \mathbf{B}_{g s 1}+\mathbf{B}_{g s 2}^{T} \mathbf{S}_{e} \mathbf{B}_{g s 2}\right)
\end{aligned}
$$

where $\underset{2 \times 20}{\mathbf{B}_{g b}}, \underset{2 \times 20}{\mathbf{B}_{g s 1}}$ and $\underset{2 \times 20}{\mathbf{B}_{g s 2}}$ are the strain-displacement matrices for geometric stiffness matrix, and for a give node $i$ :

$\mathbf{B}_{g b 1_{i}}=\left[\begin{array}{ccccc}\frac{\partial N_{i}}{\partial x} & 0 & 0 & 0 & 0 \\ \frac{\partial N_{i}}{\partial y} & 0 & 0 & 0 & 0\end{array}\right], \quad \mathbf{B}_{g b 2_{i}}=\left[\begin{array}{ccccc}0 & \frac{\partial N_{i}}{\partial x} & 0 & 0 & 0 \\ 0 & \frac{\partial N_{i}}{\partial y} & 0 & 0 & 0\end{array}\right], \quad \mathbf{B}_{g b 3_{i}}=\left[\begin{array}{ccccc}0 & 0 & \frac{\partial N_{i}}{\partial x} & 0 & 0 \\ 0 & 0 & \frac{\partial N_{i}}{\partial y} & 0 & 0\end{array}\right]$

and

$$
\mathbf{B}_{g s 1_{i}}=\left[\begin{array}{ccccc}
0 & 0 & 0 & \frac{\partial N_{i}}{\partial x} & 0 \\
0 & 0 & 0 & \frac{\partial N_{i}}{\partial y} & 0
\end{array}\right], \quad \mathbf{B}_{g s 2_{i}}=\left[\begin{array}{ccccc}
0 & 0 & 0 & 0 & \frac{\partial N_{i}}{\partial x} \\
0 & 0 & 0 & 0 & \frac{\partial N_{i}}{\partial y}
\end{array}\right] .
$$

$\mathbf{S}_{e}$ is the rearranged in-plane stress resultant matrix in the $e$-th element written as:

$$
\mathbf{S}_{e}=\left[\begin{array}{cc}
N_{x} & N_{x y} \\
N_{x y} & N_{y}
\end{array}\right]
$$

with $N_{x}, N_{y}$, and $N_{x y}$ for the in-plane stress resultants, which are obtained from the standard static analysis or so-called prebuckling analysis. In the present study, they are structural responses of the studied laminated plates subject to uniform two end shortening displacements, which will be explained further in the numerical study section.

\subsection{Predicting variations in buckling performance}

With the equations for a deterministic analysis at hand, it is possible now to derive the predictive formulation for buckling uncertainty quantification. 
We initially define an $m$-dimensional random vector $\boldsymbol{b}=\left\{b_{1}, b_{2}, \cdots, b_{m}\right\}^{T}$ to represent the set of random variables, e.g. material properties and fibre path parameters in the present study. As the buckling eigenvalue is a function of the constituent material properties and fibre angle as indicated in Eq. (2), the presence of variations in these input parameters eventually leads the buckling eigenvalue to be a stochastic function. It is well known that an arbitrary stochastic function, $\varphi(\boldsymbol{b})$, can be approximated via a Taylor series expansion as

$$
\varphi(\boldsymbol{b})=\varphi(\overline{\boldsymbol{b}})+\epsilon \sum_{r=1}^{m} \frac{\partial \varphi(\overline{\boldsymbol{b}})}{\partial b_{r}} \delta b_{r}+\epsilon^{2} \frac{1}{2} \sum_{r=1}^{m} \sum_{s=1}^{m} \frac{\partial^{2} \varphi(\overline{\boldsymbol{b}})}{\partial b_{r} \partial b_{s}} \delta b_{r} \delta b_{s}+\cdots
$$

where $\overline{\boldsymbol{b}}$ is the mean value of the random vector $\boldsymbol{b}, \delta b_{r}$ denotes the variation around mean value of the $r$-th random variable, $\frac{\partial \varphi}{\partial b_{r}}$ and $\frac{\partial^{2} \varphi}{\partial b_{r} \partial b_{s}}$ denote the first- and second-order partial derivatives of $\varphi(\boldsymbol{b})$ with respect to the $r$ - or/and $s$-th variables, respectively, and $\epsilon$ is a scalar representing a given small perturbation. In addition, given the approximation for $\varphi(\boldsymbol{b})$ in Eq. (13), its statistics in terms of mean value and variance can be easily calculated from the following equations for the second-order approximation. According to the definition of the mean value, $\int_{-\infty}^{+\infty} \varphi(\boldsymbol{b}) p(\boldsymbol{b}) d \boldsymbol{b}$, substituting Eq. (13) and using the definitions $\int_{-\infty}^{+\infty} p\left(b_{r}\right) d b_{r}=1, \int_{-\infty}^{+\infty} \delta b_{r} p\left(b_{r}\right) d b_{r}=0$ and $\int_{-\infty}^{+\infty} \delta b_{r} \delta b_{s} p\left(b_{r}\right) p\left(b_{s}\right) d b_{r} d b_{s}=C O V\left(b_{r}, b_{s}\right)$, then the mean value of $\varphi(\boldsymbol{b})$ is,

$$
E[\varphi(\boldsymbol{b})]=[\varphi(\overline{\boldsymbol{b}})]+\frac{1}{2} \sum_{r}^{m} \sum_{s}^{m}\left[\frac{\partial^{2} \varphi(\overline{\boldsymbol{b}})}{\partial b_{r} \partial b_{s}}\right] \cdot \operatorname{COV}\left(b_{r}, b_{s}\right) .
$$


Similarly, based on its definition $\int_{-\infty}^{+\infty}\left\{\varphi\left(\boldsymbol{b}_{r}\right)-E[\varphi(\boldsymbol{b})]\right\}\left\{\varphi\left(\boldsymbol{b}_{s}\right)-E[\varphi(\boldsymbol{b})]\right\} p(\boldsymbol{b}) d \boldsymbol{b}$, the variance is found:

$$
\begin{aligned}
\operatorname{COV}([\varphi(\boldsymbol{b})],[\varphi(\boldsymbol{b})]) \approx & \sum_{r, s}^{m}\left[\frac{\partial \varphi(\overline{\boldsymbol{b}})}{\partial b_{r}}\right]\left[\frac{\partial \varphi(\overline{\boldsymbol{b}})}{\partial b_{s}}\right] \cdot \operatorname{COV}\left(b_{r}, b_{s}\right) \\
& +\frac{1}{4} \sum_{r, s, t, w}^{m}\left[\frac{\partial^{2} \varphi(\overline{\boldsymbol{b}})}{\partial b_{r} \partial b_{s}}\right]\left[\frac{\partial^{2} \varphi(\overline{\boldsymbol{b}})}{\partial b_{t} \partial b_{w}}\right] E\left[b_{r} b_{s} b_{t} b_{w}\right] .
\end{aligned}
$$

with $r, s, t, w$ denote the corresponding random variable in $\boldsymbol{b}$. Therefore, it is crucial to obtain the first- and second-order partial derivatives of the stochastic function with respect to the random variables to estimate its mean value and covariance from Eqs. (14) and (15), respectively.

\subsubsection{Statistics of critical buckling load or coefficient}

When combining Eq. (13) with a finite element representation of the linear buckling analysis in Eq. (2), a stochastic form of the eigenvalue problem can be defined, which is a popular uncertainty quantification technique featured with relatively small computational cost and promising accuracy. In the present study, the second-order approximation has been found to be sufficient, whilst higher-order approximations may be used to describe stochastic functions that are highly nonlinear functions of the underlying random variables.

Taking the partial derivative of Eq. (2) with respect to the $r$-th parameter yields the relation,

$$
\left(\frac{\partial \mathbf{K}}{\partial b_{r}}-\frac{\partial \lambda_{i}}{\partial b_{r}} \mathbf{K}_{g}-\lambda_{i} \frac{\partial \mathbf{K}_{g}}{\partial b_{r}}\right) \boldsymbol{v}_{i}+\left(\mathbf{K}-\lambda_{i} \mathbf{K}_{g}\right) \frac{\partial \boldsymbol{v}_{i}}{\partial b_{r}}=\mathbf{0}
$$

The first partial derivative of $\lambda_{i}$ may now be found by premultiplying Eq. (16) by the transpose of the left-hand eigenvector $\mathbf{v}_{i}$, then substituting Eq. 
(2) into the results and finally solving for the partial derivative, such that,

$$
\frac{\partial \lambda_{i}}{\partial b_{r}}=\left[\boldsymbol{v}_{i}^{T}\left(\frac{\partial \mathbf{K}}{\partial b_{r}}-\lambda_{i} \frac{\partial \mathbf{K}_{g}}{\partial b_{r}}\right) \boldsymbol{v}_{i}\right] /\left(\boldsymbol{v}_{i}^{T} \mathbf{K}_{g} \boldsymbol{v}_{i}\right)
$$

Similarly, the second order partial derivative of the eigenvalue $\lambda_{i}$ may be found by differentiating Eq. (16) with respect to the $s$-th parameter, premultiplying by the transpose of the left-hand eigenvector $\boldsymbol{v}_{i}$, making use of Eq. (2) and then solving for the second partial derivative:

$$
\begin{aligned}
\frac{\partial^{2} \lambda_{i}}{\partial b_{r} \partial b_{s}}= & {\left[\boldsymbol{v}_{i}^{T}\left(\frac{\partial^{2} \mathbf{K}}{\partial b_{r} \partial b_{s}}-\frac{\partial \lambda_{i}}{\partial b_{r}} \frac{\partial \mathbf{K}_{g}}{\partial b_{s}}-\frac{\partial \lambda_{i}}{\partial b_{s}} \frac{\partial \mathbf{K}_{g}}{\partial b_{r}}-\lambda_{i} \frac{\partial^{2} \mathbf{K}_{g}}{\partial b_{r} \partial b_{s}}\right) \boldsymbol{v}_{i}\right.} \\
& +\boldsymbol{v}_{i}^{T}\left(\frac{\partial \mathbf{K}}{\partial b_{r}}-\frac{\partial \lambda_{i}}{\partial b_{r}} \mathbf{K}_{g}-\lambda_{i} \frac{\partial \mathbf{K}_{g}}{\partial b_{r}}\right) \frac{\partial \boldsymbol{v}_{i}}{\partial b_{s}} \\
& \left.+\boldsymbol{v}_{i}^{T}\left(\frac{\partial \mathbf{K}}{\partial b_{s}}-\frac{\partial \lambda_{i}}{\partial b_{s}} \mathbf{K}_{g}-\lambda_{i} \frac{\partial \mathbf{K}_{g}}{\partial b_{s}}\right) \frac{\partial \boldsymbol{v}_{i}}{\partial b_{r}}\right] /\left(\boldsymbol{v}_{i}^{T} \mathbf{K}_{g} \boldsymbol{v}_{i}\right)
\end{aligned}
$$

To compute the second order derivative of the eigenvalue, $\lambda_{i}$, several additional elements are required, including the second order derivatives of $\mathbf{K}$ and $K_{g}$. The first order derivative of the eigenvector, while the calculation of $\partial \boldsymbol{v}_{i} / \partial b_{r}$, is also needed, and is less straightforward to compute than $\frac{\partial^{2} \mathbf{K}}{\partial b_{r} \partial b_{s}}$ and $\frac{\partial^{2} \mathbf{K}_{\mathbf{g}}}{\partial b_{r} \partial b_{s}}$. Several methods, including the Nelson method (Nelson, 1976), the modal method (Fox and Kapoor, 1968), and the modified modal method (Wang, 1991), are available to calculate $\partial \boldsymbol{v}_{i} / \partial b_{r}$. In the present study, the modified modal method is adopted as a compromise between accuracy and computational efficiency (Sutter et al., 1988). It uses a linear combination of a given number of eigenvectors as shown in Eq. (19).

$$
\frac{\partial \boldsymbol{v}_{i}}{\partial b_{r}}=\mathbf{K}^{-1}\left(\frac{\partial \lambda_{k}}{\partial b_{r}} \mathbf{K}_{g}-\frac{\partial \mathbf{K}}{\partial b_{r}}+\lambda_{k} \frac{\partial \mathbf{K}_{g}}{\partial b_{r}}\right) \boldsymbol{v}_{k}+\sum_{j=1} c_{k j} \boldsymbol{v}_{j}
$$

where the coefficients $c_{k j}$ are defined as:

$$
c_{k j}=\lambda_{k}\left[\boldsymbol{v}_{j}^{T}\left(\frac{\partial \mathbf{K}}{\partial b_{r}}-\lambda_{k} \frac{\partial \mathbf{K}_{g}}{\partial b_{r}}\right) \boldsymbol{v}_{k}\right] /\left[\lambda_{j}\left(\lambda_{k}-\lambda_{j}\right) \boldsymbol{v}_{j}^{T} \mathbf{K}_{g} \boldsymbol{v}_{j}\right], \quad k \neq j(
$$




$$
c_{k k}=-\frac{1}{2} \boldsymbol{v}_{k}^{T} \frac{\partial \mathbf{K}_{g}}{\partial b_{r}} \boldsymbol{v}_{k}
$$

149

Note that $\boldsymbol{v}_{k}$ in Eq. (21) is normalized with $\mathbf{K}_{g}$, i.e. $\boldsymbol{v}_{k}^{T} \mathbf{K}_{g} \boldsymbol{v}_{k}=1$.

In the present study, the uncertainties in material properties and fibre paths are of interest thereby treated as random variables. For the material properties, the basic variables are defined as, $\boldsymbol{b}=\left\{E_{1 k}, E_{2 k}, \nu_{12 k}, \nu_{23 k}, G_{13 k}\right\}^{T}, k=$ $1,2, \cdots, N P$. The first- and second-order partial derivatives of $\mathbf{K}$ are then:

$$
\begin{gathered}
\frac{\partial \mathbf{K}}{\partial b_{r}}=\sum_{e=1}^{N E} \mathbf{B}^{T} \frac{\partial \hat{\mathbf{D}}_{e}}{\partial b_{r}} \mathbf{B}=\sum_{e=1}^{N E} \sum_{k=1}^{N P} \mathbf{B}^{T} \mathbf{L}_{e k}^{T} \frac{\partial \hat{\mathbf{D}}_{e k}}{\partial b_{r}} \mathbf{L}_{e k} \mathbf{B} \\
\frac{\partial^{2} \mathbf{K}}{\partial b_{r} \partial b_{s}}=\sum_{e=1}^{N E} \mathbf{B}^{T} \frac{\partial^{2} \hat{\mathbf{D}}_{e}}{\partial b_{r} \partial b_{s}} \mathbf{B}=\sum_{e=1}^{N E} \sum_{k=1}^{N P} \mathbf{B}^{T} \mathbf{L}_{e k}^{T} \frac{\partial^{2} \hat{\mathbf{D}}_{e k}}{\partial b_{r} \partial b_{s}} \mathbf{L}_{e k} \mathbf{B}
\end{gathered}
$$

where for $\frac{\partial \hat{\mathbf{D}}_{e k}}{\partial b_{r}}$ it is required to calculate $\frac{\partial \mathbf{C}_{1 k}}{\partial b_{r}}$ and $\frac{\partial \mathbf{C}_{2 k}}{\partial b_{r}}$ as functions of the material properties as indicated in Eq. (5). In this way, uncertainties in the material properties can be propagated to the buckling eigenvalues.

It is also required to calculate the first- and second-order partial derivatives of $\mathbf{K}$ with respect to fibre tow path parameters in Eq. (1) to propagate uncertainties associated with variations in fibre paths. As the fibre path directions are assumed to vary linearly, uncertainty in $\mathbf{K}$ will propagate through the parameters $\boldsymbol{b}=\left\{\phi_{k}, T_{0 k}, T_{1 k}\right\}^{T}, k=1,2, \cdots, N P$. The partial derivatives of $\mathbf{K}$ with respect to $\boldsymbol{b}$ are calculated, therefore, as:

$$
\begin{aligned}
\frac{\partial \mathbf{K}}{\partial b_{r}} & =\sum_{e=1}^{N E} \sum_{k=1}^{N P} \frac{\partial \mathbf{k}_{e}}{\partial \theta_{e k}} \frac{\partial \theta_{e k}}{\partial b_{r}} \\
& =\sum_{e=1}^{N E} \sum_{l=1}^{N P}\left[\mathbf{B}^{T}\left(\frac{\partial \mathbf{L}_{e k}^{T}}{\partial \theta_{e k}} \mathbf{D}_{e k} \mathbf{L}_{e k}+\mathbf{L}_{e k}^{T} \mathbf{D}_{e k} \frac{\partial \mathbf{L}_{e k}}{\partial \theta_{e k}}\right) \frac{\partial \theta_{e k}}{\partial b_{r}} \mathbf{B}\right]
\end{aligned}
$$


and

$$
\begin{aligned}
\frac{\partial^{2} \mathbf{K}}{\partial b_{r} \partial b_{s}} & =\sum_{e=1}^{N E} \sum_{k=1}^{N P}\left[\frac{\partial^{2} \mathbf{k}_{e}}{\partial \theta_{e k}^{2}} \frac{\partial \theta_{e k}}{\partial b_{r}} \frac{\partial \theta_{e k}}{\partial b_{s}}+\frac{\partial \mathbf{k}_{e}}{\partial \theta_{e k}} \frac{\partial^{2} \theta_{e k}}{\partial b_{r} \partial b_{s}}\right] \\
& =\sum_{e=1}^{N E} \sum_{k=1}^{N P}\left[\mathbf{B}^{T}\left(\frac{\partial^{2} \mathbf{L}_{e k}^{T}}{\partial \theta_{e k}^{2}} \mathbf{D}_{e k} \mathbf{L}_{e k}+\mathbf{L}_{e k}^{T} \mathbf{D}_{e k} \frac{\partial^{2} \mathbf{L}_{e k}}{\partial \theta_{e k}^{2}}\right) \frac{\partial \theta_{e k}}{\partial b_{r}} \frac{\partial \theta_{e k}}{\partial b_{s}} \mathbf{B}\right]
\end{aligned}
$$

\section{Results and discussion}

In this research, a twelve-layer VAT composite laminate studied in (Gürdal et al., 2008) has been adopted to understand its stochastic mechanical behaviours. It is made up of AS4 carbon fibres and a 3501-6 epoxy matrix with 
material properties: $E_{1}=181 G P a, E_{2}=8.96 G P a, \nu_{12}=0.3, \nu_{23}=0.28$ and $G_{13}=7.2 G P a$. It has a total thickness of $h=1.524 \mathrm{~mm}$ with all plies having an identical thickness of $0.127 \mathrm{~mm}$, and it is square with length and width of $a=0.254 \mathrm{~m}$. A uniform end shortening displacement is applied to the structure along $x$ direction. Following (Gürdal et al., 2008), two types of boundary conditions recognized as typical ones are considered, which include one with translation constrained in the y-direction on the transverse edge (denoted as Case I shown in Figure 3) and another with the transverse edge free to deform (denoted as Case II shown in Figure 3). Several numerical studies have been conducted to understand the variations in VAT plates performances with the use of the proposed uncertainty quantification method. In additional to buckling eigenvalue, a further buckling measure, namely, the critical buckling coefficient, which is related to critical buckling strength in the form of load normalized by $a^{2} /\left(E_{1} h^{3}\right)$ for better indicating of the enhancement comparing with a straight fibre laminate is used and calculated as:

$$
K_{c r}=\lambda N_{x}^{c r} \frac{a^{2}}{E_{1} h^{3}}
$$

where $N_{x}^{c r}$ is the critical loading in terms of axial stress resultant. For the Case I panel, $N_{x}^{c r}$ is the same as $N_{x}$ because it is constant over the panel, while $N_{x}$ varies in $y$-direction and $N_{x}^{c r}$ is an average critical load calculated by $\bar{N}_{x}^{c r}=\frac{1}{b} \int_{-h / 2}^{h / 2} N_{x}(y) d y$ (Gürdal et al., 2008) for Case II. Eq. (25) is approximated again using Taylor series expansion with its first- and secondorder derivatives given in Eq. (26). Thus, the outcomes from PSFE are the 
required basic elements to predict statistics of the critical buckling coefficient.

$$
\begin{aligned}
\frac{\partial K_{c r}}{\partial b_{r}} & =\left(\frac{\partial \lambda}{\partial b_{r}} N_{x}^{c r}+\lambda \frac{\partial N_{x}^{c r}}{\partial b_{r}}\right) \frac{a^{2}}{E_{1} h^{3}} \\
\frac{\partial^{2} K_{c r}}{\partial b_{r} \partial b_{s}} & =\left(\frac{\partial^{2} \lambda}{\partial b_{r} \partial b_{s}} N_{x}^{c r}+\frac{\partial \lambda}{\partial b_{r}} \frac{\partial N_{x}^{c r}}{\partial b_{s}}+\frac{\partial \lambda}{\partial b_{s}} \frac{\partial N_{x}^{c r}}{\partial b_{r}}+\lambda \frac{\partial^{2} N_{x}^{c r}}{\partial b_{r} \partial b_{s}}\right) \frac{a^{2}}{E_{1} h^{3}}
\end{aligned}
$$

\subsection{Model validation}

\subsubsection{Finite element analysis}

Table 1 illustrates the first five buckling eigenvalues of the investigated VAT plates. The results obtained using the in-house finite element implementation are compared with those from ANSYS. The laminate has been discretized into a mesh of 20 by 20 elements. In ANSYS, the shell181 element, which is a four node rectangular shell element and suitable for analyzing thin to moderately-thick shell structures, is chosen as the underlying theory is the same as the one used in the present study. Elemental fibre angles from Eq. (1) are imported into the finite element model in ANSYS. For the two panels, two types of layup with one for single player and another with 12 layers have been considered. For a single layer laminate, the fibre path is defined by $0^{\circ}<0^{\circ} \mid 50^{\circ}>$ for Case I and $90^{\circ}<0^{\circ} \mid 75^{\circ}>$ for Case II. For the multilayer laminates, Case I panel features with fibre paths of $0^{\circ} \pm<0^{\circ} \mid 50^{\circ}>_{3 s}$ and Case II of $90^{\circ} \pm<0^{\circ} \mid 75^{\circ}>_{3 s}$. Eigenvalues under the applied displacement have been calculated by both ANSYS and in-house code, and results for the first five modes are listed in the table. The comparisons show that the in-house code match excellently with those from ANSYS. In addition, the critical buckling loads for the two multilayer laminates from the in-house are compared with those reported in (Gürdal et al., 2008), with a load of 1.41 from the former, representing a prediction around $2 \%$ less than 
that reported in (Gürdal et al., 2008) of 1.44 for Case I panel. For Case II panel, the maximum normalized value of the critical load is 3.07 , which is only about $2 \%$ less that the reference value of 3.14 in (Gürdal et al., 2008). In all the cases, the critical buckling loads are slightly smaller than those found in Gürdal et al. (2008), where the buckling problem was solved by the Ritz method, which results in a slight overestimate of the actual buckling load as a finite set of terms are taken. Hence, the implementation is proven to provide promising predictions.

\subsubsection{Uncertainty quantification}

Before applying PSFE to study the probabilistic mechanical behaviours of VAT composites, its accuracy and efficiency are investigated through comparisons with outcomes from MCS. A simulation with 5000 samples has been performed to estimate the buckling statistics in the presence of uncertainties in material properties and fibre paths. Statistics of the buckling eigenvalues and the critical buckling coefficients are investigated. Although the perturbation method is independent of the type of probability distributions describing the input random variables, in principle, Normal distributions are usually considered to produce more accurate estimates due to their symmetry properties. For MCS, material properties are assumed to be Normally distributed variables, and fibre tow path parameters uniformly distributed. Two special cases are considered. In the first case, uncertainties are limited to variability in the material properties, with a coefficient of variation of $10 \%$. In the second case, variations in the fibre path with its parameters distributed with $\left[-5^{\circ},+5^{\circ}\right]$ are investigated. Results are presented in Tables 2 and 3. Both mean value and standard deviation predictions from 
the proposed perturbation-based method are sufficiently close to those from the MCS. It indicates that PSFE works well when having these practical uncertainties.

It is well known that PSFE performs well for small variations, but its accuracy is less assured for large variations when stochastic functions are strongly nonlinear with respect to the input random variables. It is crucial to understand the feasible regions to be confident to use PSFE for more advanced studies. It is not straightforward to qualify the extent of nonlinearity of the buckling eigenvalues and the critical buckling coefficients with respect to material properties and fibre tow path. Further numerical studies have been conducted to determine the acceptable ranges of the coefficient of variations in these two sets of random variables for accurately predicting buckling statistics for VAT laminates. Outcomes of PSFE studies are given in Figures 4 and 5. Considering a 'good' approximation as one giving less than a 5\% discrepancy from the exact result, i.e. from MCS here, it appears that estimates of the coefficients of variation for the critical buckling loads can be accepted as long as coefficients of variation in the material properties are less than 0.3 (or 30\%), whilst smaller variations in fibre tow path parameters, i.e. $\pm 7.5^{\circ}$, should be considered reasonable for PSFE. This can be partially explained by the fact that the exact Taylor series expansion describing the critical buckling coefficient with respect to material properties is predominantly controlled by constant and linear terms. Furthermore, it may be explained from the constitutive model which is a linear function of Young's modulus and the shear modulus, while a nonlinear function of the Poisson's ratios. With the increase of variability in the Poisson's ratios, the 
terms including $1 /(1-2 \nu)$ have the possibility (although improbable) to take values approaching infinity, with consequential loss of accuracy in predicting the statistics of the buckling estimates. In contrast, nonlinear terms related to fibre tow paths have significant contributions in the terms omitted from the truncated Taylor series expansion describing the buckling measure, due to the nature of fibre tow angle's contribution through the terms $\cos \theta$ and $\sin \theta$, which are infinitely differentiable, i.e. the existence of significant higher-order nonlinear terms. As a conclusion, it is seen in this example that the second-order perturbation method can provide sufficiently accurate estimates for buckling statistics given relatively large uncertainties in the material properties. Whilst the second-order perturbation method cannot necessarily provide satisfactory results for medium to large coefficients of variation in the input random variables for fibre angles, it can provide sufficiently accurate estimates when the fluctuations are in the range $\pm 7.5^{\circ}$, which is the most commonly reported variability in fibre misalignment made from AFP, e.g. (Potter et al., 2008). Therefore, it is hypothesised that PSFE can be confidently used to investigate the uncertainties in these two sets of random variables for understanding practical and realistic variations in VAT composite plates.

\subsection{Effect of material properties}

For the two cases, statistics of buckling measures have been calculated parametrically with various angles of $T_{1}$ from $0^{\circ}$ to $90^{\circ}$ while keeping the other two parameters $\phi$ and $T_{0}$ unchanged, i.e. $\phi=0^{\circ}$ and $T_{0}=0^{\circ}$ for Case I panel, while $\phi=90^{\circ}$ and $T_{0}=0^{\circ}$ for Case II. All the five independent material properties, i.e. $E_{1}, E_{2}, \nu_{12}, G_{12}$ and $G_{23}$, have been considered as 
random variables with coefficient of variation of 0.1 or $10 \%$. Eqs.(17) and (18) are used to obtain ingredients to calculate the mean value and the standard deviation of eigenvalue from Eqs (14) and (15), respectively, while Eq.(26) is called for the critical buckling coefficient. For each angle of $T_{1}$, statistics of these two buckling measures have been calculated for the two cases. The probabilistic buckling analysis results are reported in terms of statistics of buckling eigenvalues and critical buckling coefficients in Figures 6 and 7 for Cases I and II, respectively.

For Case I, the peak (or the maximum) eigenvalue appears around $T_{1}=$ $80^{\circ}$, which is different from the angle $T_{1}=50^{\circ}$ for the maximum of critical buckling coefficient, while the minima of the eigenvalue standard deviation and coefficient of variation occur when the critical buckling coefficient reaches its maximum. Figure $6 \mathrm{a}$ shows that the coefficients of variation for eigenvalue vary from $0.2 \%$ to $4.5 \%$, while the coefficients of variation for critical buckling coefficient are around $8 \%$ with slight dependence with respect to the variation of $T_{1}$ shown in Figure $6 \mathrm{~b}$. There is clear evidences that variation in the critical buckling coefficient increases when the structure shows better buckling performance, while no such a clear trend is shown for the variation in the corresponding eigenvalues. It is understood that the enhancement of buckling performance of Case I panel is largely due to a favourable distribution of the transverse stress resultant $N_{y}=A_{11} / A_{12} N_{x}$ at the panel edges along the length of the panel, where $A_{11}$ and $A_{12}$ are terms of the membrane stiffness matrix and $N_{x}$ is the x-direction in-plane stress resultant with constant value. Hence, the coefficient of variation in the eigenvalue becomes smaller when the structure becomes stiffer with the plate, therefore, achiev- 
ing a higher buckling strength. In contrast, the coefficient of variation in the critical buckling factor, attains its maximum value at almost the same point at which the mean value reaches its maximum. This is partially due to the fact that the critical buckling coefficient is a function of $\lambda N_{x}$ which may be dominated by variations in $N_{x}$, as its mean value remains as a constant and its variation is only affected by uncertainties in material properties.

Similarly, statistics for the two buckling measures are given in Figure 7 for the Case II panel. It is observed that the variations in the eigenvalue and the critical buckling coefficient are linearly correlated to the input variations. For instance, the majority of the coefficients of variation of eigenvalue are around $10 \%$ with a $10 \%$ input variations in the material properties as illustrated in Figure 7a, while the coefficient of variation for the critical buckling coefficient gradually increases with $T_{1}$ before reaching its maximum shown in Figure 7b. Again, the peaks in the eigenvalue mean value and standard deviation occur when $T_{1} \approx 55^{\circ}$ while the peak for the critical buckling coefficient appears when $T_{1}$ is around $75^{\circ}$. This is potentially caused by the fact that the eigenvalue only reflects the buckling occurrence in terms of the displacement, while the critical buckling coefficient measures the buckling strength by transforming the displacement based strength into the load. In addition, it is understood that the reason for the substantially improved buckling response characteristics is due to a favourable distribution of the applied load across the panel width as a function of the $y$ coordinate, which is different from the one observed for the Case I panel. $N_{x y}$ and $N_{y}$ are zero over the panel, while $N_{x}(y)=2 u_{0}\left[A_{11}-A_{12}^{2} / A_{22}\right] / a$ with $A_{11}, A_{12}$ and $A_{22}$ are the terms of in-plane stiffness matrix, $u_{0}$ is the applied uniform displacement, 
and $a$ is the panel length. The variation of the eigenvalue is directly linked to the variation in the stiffness, and the same magnitude of the coefficient of variation is thus observed in the eigenvalue as the input. In addition, the critical buckling load factor is calculated through $\lambda \int N_{x}(y) d y / b$, which indicates that its variation is around the square sum of the corresponding values for $\lambda$ and $N_{x}$. For instance, the maximum coefficient of variation of the critical buckling coefficient is around $\sqrt{2} \times 10 \% \approx 14.1 \%$. In addition, the slight discontinuities in the curves for coefficient of variation is caused by the change of buckling mode shape when $T_{1}$ increases from $73^{\circ}$ to $74^{\circ}$ as shown in Figure 8. The first two buckling mode shapes swap their positions, i.e. the first mode in $T_{1}=73^{\circ}$ becomes the second mode in $T_{1}=74^{\circ}$. Due to the fact that the eigenvalues of these two first modes are very close, there is no apparent discontinuity observed on the curves of mean values. However, the swapping of mode shape leads to a jump in the first-order partial derivatives with respect to material properties. For instance, Figure 9 gives the first-order derivatives of the first two lowest eigenvalues and critical buckling coefficients with respect to the longitudinal Young's modulus, $E_{1}$, for $T_{1}$ changing from $69^{\circ}$ and $79^{\circ}$. The derivatives are discontinued between $73^{\circ}$ and $744^{\circ}$ due the swapping of mode shapes. It finally reflects on the discontinuities in the curves of coefficient of variation as they are functions of the first order partial derivatives.

\subsection{Effect of fibre tow angle}

In this section, statistics of buckling performance measures are investigated with considerations of uncertainties in fibre tow paths for the two panels. Again, $\phi$ and $T_{0}$ keep unchanged while $T_{1}$ varies from $0^{\circ}$ to $90^{\circ}$. The 
uncertainties in fibre tow paths are considered through the three parameters $\phi, T_{0}$ and $T_{1}$. In this study,they are treated as uniform distribution variables with fluctuations of $\pm 5^{\circ}$. For each $T_{1}$, mean values and standard deviations for the two buckling measures are calculated from Eqs. (14) and (15), respectively. They are then shown in Figures 10 and 12 for Cases I and II, respectively.

For the eigenvalue curves of Case I, the standard deviations in the upper panel of Figure 10 show almost the same trend as the mean value with increasing $T_{1}$. However, the standard deviation reaches its maximum at $T_{1} \approx 65^{\circ}$, which is different to the mean value. In addition, as changes in the standard deviations differ from those of the mean values, the coefficients of variation vary from 0 to $4 \%$ with the increase of $T_{1}$. In general, the coefficient of variation follows a similar pattern to the standard deviation. According to Eq. (15), the first order derivatives are major contributors to the standard deviation. Hence, these observations are confirmed by the plots of the first order derivatives of the eigenvalue with respect to the three parameters, $\phi$, $T_{0}$ and $T_{1}$ as given in Figure 11. The change of $T_{1}$ almost linearly affects the positive contributions of these three parameters before reaching its maximum for upper panel figure for eigenvalues. For the statistics describing the critical buckling coefficient shown in the lower panel of Figure 10, the plot of the standard deviation shows a more complicated trend. Figure 10b indicates that the standard deviation reaches a local maximum around $T_{1}=25^{\circ}$ before it reaches its local minimum around $T_{1}=50^{\circ}$ when the mean value attains its maximum, but the standard deviation then increases till $T_{1} \approx 80^{\circ}$. A close inspection of the first order derivatives of the critical buckling coefficient 
shown in Figure 11b explains the fluctuation as the three parameters switch from positive contributions to the standard deviation to negative contributions with the increasing of $T_{1}$. The coefficient of variation shows a similar trend as the standard deviation. Again, it is not straightforward to link the variation of coefficient of variation with the change of fibre angle path in terms of $T_{1}$ here. The transverse stress resultant $N_{y}=A_{11} / A_{12} N_{x}$ indicates that the variation in eigenvalue is closely linked with changes in the stiffness as reflected in $N_{x}$ but slightly affected by $A_{11} / A_{12}$. Hence, the eigenvalue coefficients of variation and critical buckling coefficient become smaller when the structure becomes stiffer, thereby displaying a higher buckling strength. For the eigenvalue plots of Case II panel, the standard deviations in Figure 12a show that they become larger before reducing in magnitude to reach a local minimum when the eigenvalue displays a maximum value with the change of $T_{1}$ from 0 to $90^{\circ}$. This trend appears on the plot of the coefficient of variation for the buckling eigenvalue in the lower diagram in Figure 12a. Similarly, the stochastic analysis confirms that the buckling eigenvalue reaches its maximum before swapping buckling mode shape as a jump on the critical buckling coefficient curve is found, which shows similar phenomenon as the one with uncertainties in material properties. This is further confirmed by the plot of the first order derivatives of eigenvalue and critical buckling coefficient in Figure 13. To show clearly the discontinuities of the buckling statistics caused by mode switching, the first order partial derivatives of the eigenvalues and critical buckling coefficients for the first two modes are given in Figure 14, where it can be seen that the first order partial derivatives of the lowest eigenvalues are swapped. According to Eq. (15), the first order 
derivatives are major contributors of the standard deviation. For the statistics of critical buckling coefficient shown in the lower panel of Figure 12, the curve of standard deviation shows almost the same trend as the mean value with the increase of $T_{1}$. This leads to a plateau for the curve of the coefficient of variation. Although there is no evident link between the increase of $T_{1}$ and the variation of the coefficient of variation of the buckling measures, the varying of transverse stress resultant $N_{x}(y)=2 u_{0}\left[A_{11}-A_{12}^{2} / A_{22}\right] / a$ in $y$-direction indicates that the variation in eigenvalue is originated from the changes in the stiffness as $A_{11}, A_{12}$ and $A_{22}$ are functions of fibre tow path.

\subsection{Effect of boundary conditions}

As it has been well recognized, boundary conditions have a significant influence on the buckling performance of VAT composite panels. For example, Case I panel with restricted translation in the y-direction may have a maximum critical buckling coefficient of around 1.41 with fibre tow path defined by $0^{\circ} \pm<0^{\circ} \mid 50^{\circ}>_{3 s}$, whilst the Case II panel with free deformation on the transverse edges has a higher critical buckling coefficient of 3.07 with a fibre tow path of $90^{\circ} \pm<0^{\circ} \mid 75^{\circ}>_{3 s}$. Their buckling statistics with uncertainties in either material properties or fibre tow path also display clear differences as shown in Figures 6 and 7, and 10 and 12, respectively. For instance, in the presence of uncertainties in material properties, the variations in eigenvalue in Case I have completely different trends and also magnitudes of coefficients of variation when the angle $T_{1}$ is varied. Figure 15 shows the sensitivities of the critical buckling coefficients with respect to variation in each material property for the two panels with their optimal design given in (Gürdal et al., 2008). The buckling statistics of Case I panel are mostly affected by $\nu_{23}$, 
while Case II panel is most sensitive to $\nu_{12}$. In terms of the influence of each material property, it can be seen that the Case I panel is positively linked with $E_{1}, E_{2}, \nu_{23}$, and $G_{13}$, but $\nu_{12}$ has a negative contribution to its variation. While $E_{1}, \nu_{12}$, and $G_{13}$ have positive effects on the buckling statics of Case II panel, $E_{2}$ and $\nu_{23}$ show negative correlations. It appears that these characteristics are due to changes in the boundary conditions. For variations in buckling performance measures arising from uncertainties in fibre tow path, no significant difference between the two boundary conditions is observed.

\section{Conclusions}

In this paper, the widely used uncertainty quantification method, namely perturbation-based stochastic finite element method, is adopted to investigate the variability in mechanical performance of variable angle tow composite plates, which are tailored to have an enhanced buckling performance. Variations in buckling performance arising from uncertainties in material properties and fibre tow path are investigated and measured by its first two moments, namely mean value and standard deviation. Detailed formulations for predicting buckling statistics are derived and given to conduct a perturbation-based stochastic finite element analysis. As the primary objective of this work is to understand the variations in buckling performance, relatively simple VAT composite plates with the use of a linear variation of the fibre tow path are studied. However, the formulae can be easily extended to analyse VAT plates with more complicated fibre tow paths.

The use of PSFE to quantify uncertainties in buckling performance measures including eigenvalue and critical buckling coefficient has been verified 
first through comparing statistics against corresponding values from MCS. The comparisons show that PSFE can predict the variations in buckling measures with sufficient accuracy, while the computational cost can be significantly reduced. PSFE can successfully propagate uncertainties in material properties with a coefficient of variation of 0.3 , and it is also applicable to quantify uncertainties in fibre tow path, which have fluctuations as large as about $\pm 10^{\circ}$. Therefore, PSFE can deal with these two types of uncertainties with a sufficiently wide range. With this confidence, the method is further applied to understand the stochastic mechanical behaviour of VAT composite plates. Results show that buckling statistics in terms of mean value and standard deviation or coefficient of variation are significant functions of uncertainties in fibre tow paths, and should not be ignored. Uncertainties in buckling measures arising from variations in material properties are positively correlated as statistics reaches their maxima while maximal buckling performances are achieved. In contrast, statistics of buckling measures have their minima while optimal buckling performance attains. These findings imply the importance to take variations in material properties into account to find robust design of VAT composite structures.

\section{References}

Bednarcyk, B. A., Aboudi, J., Arnold, S. M., 2014. The effect of general statistical fiber misalignment on predicted damage initiation in composites. Composites Part B: Engineering 66 (0), 97-108.

Belnoue, J. P. H., Mesogitis, T., Nixon-Pearson, O. J., Kratz, J., Ivanov, D. S., Partridge, I. K., Potter, K. D., Hallett, S. R., 2017. Understand- 
ing and predicting defect formation in automated fibre placement prepreg laminates. Composites Part A: Applied Science and Manufacturing 102 (Supplement C), 196-206.

Chen, N.-Z., Guedes Soares, C., 2007. Reliability assessment of post-buckling compressive strength of laminated composite plates and stiffened panels under axial compression. International Journal of Solids and Structures 44 (2223), 7167-7182.

Endruweit, A., Gommer, F., Long, A. C., 2013. Stochastic analysis of fibre volume fraction and permeability in fibre bundles with random filament arrangement. Composites Part A: Applied Science and Manufacturing 49 (0), $109-118$.

Ferreira, A. J. M. (Ed.), 2009. Analysis of Mindlin plates. Springer Netherlands, Dordrecht, pp. 161-201.

Fox, R. L., Kapoor, M. P., 1968. Rates of change of eigenvalues and eigenvectors. AIAA Journal 6 (12), 2426-2429.

Gürdal, Z., Tatting, B. F., Wu, C. K., 2008. Variable stiffness composite panels: Effects of stiffness variation on the in-plane and buckling response. Composites Part A: Applied Science and Manufacturing 39 (5), 911-922.

Hörrmann, S., Adumitroaie, A., Viechtbauer, C., Schagerl, M., 2016. The effect of fiber waviness on the fatigue life of CFRP materials. International Journal of Fatigue 90, 139-147.

Hyer, M. W., Charette, R. F., 1991. Use of curvilinear fiber format in composite structure design. AIAA Journal 29 (6), 1011-1015. 
Ijsselmuiden, S. T., Abdalla, M. M., Grdal, Z., 2010. Optimization of variable-stiffness panels for maximum buckling load using lamination parameters. AIAA Journal 48 (1), 134-143.

Kim, B. C., Weaver, P. M., Potter, K., 2014. Manufacturing characteristics of the continuous tow shearing method for manufacturing of variable angle tow composites. Composites Part A: Applied Science and Manufacturing $61,141-151$.

Lal, A., Singh, B. N., Kale, S., 2011. Stochastic post buckling analysis of laminated composite cylindrical shell panel subjected to hygrothermomechanical loading. Composite Structures 93 (4), 1187-1200.

Lal, A., Singh, B. N., Kumar, R., 2009. Effects of random system properties on the thermal buckling analysis of laminated composite plates. Computers \& Structures 87 (17), 1119-1128.

Li, J., Tian, X., Han, Z., Narita, Y., 2016. Stochastic thermal buckling analysis of laminated plates using perturbation technique. Composite Structures 139 (Supplement C), 1-12.

Lin, S. C., 2000. Buckling failure analysis of random composite laminates subjected to random loads. International Journal of Solids and Structures 37 (51), 7563-7576.

Lin, S. C., Kam, T. Y., Chu, K. H., 1998. Evaluation of buckling and first-ply failure probabilities of composite laminates. International Journal of Solids and Structures 35 (13), 1395-1410. 
Lukaszewicz, D. H. J. A., Ward, C., Potter, K. D., 2012. The engineering aspects of automated prepreg layup: History, present and future. Composites Part B: Engineering 43 (3), 997-1009.

Matveev, M., Ball, F., Jones, I., Long, A., Schubel, P., Tretyakov, M., 2017. Uncertainty in geometry of fibre preforms manufactured with automated dry fibre placement and its effects on permeability. Journal of Composite Materials, 0021998317741951.

Nelson, R. B., 1976. Simplified calculation of eigenvector derivatives. AIAA Journal 14 (9), 1201-1205.

Oñate, E., 2013. Structural analysis with the finite element method linear statics: volume 2 . beams, plates and shells. Vol. 2 of Lecture notes on numerical methods in engineering and sciences. Springer Netherlands.

Orifici, A. C., Bisagni, C., 2013. Perturbation-based imperfection analysis for composite cylindrical shells buckling in compression. Composite Structures $106,520-528$.

Potter, K., Khan, B., Wisnom, M., Bell, T., Stevens, J., 2008. Variability, fibre waviness and misalignment in the determination of the properties of composite materials and structures. Composites Part A: Applied Science and Manufacturing 39 (9), 1343-1354.

Sabido, A., Bahamonde, L., Harik, R., van Tooren, M. J. L., 2017. Maturity assessment of the laminate variable stiffness design process. Composite Structures 160, 804-812. 
Setoodeh, S., Abdalla, M. M., IJsselmuiden, S. T., Grdal, Z., 2009. Design of variable-stiffness composite panels for maximum buckling load. Composite Structures 87 (1), 109-117.

Singh, B. N., Iyengar, N. G. R., Yadav, D., 2001. Effects of random material properties on buckling of composite plates. Journal of Engineering Mechanics 127 (9), 873-879.

Sutter, T. R., Camrada, C. J., Walsh, J. L., Adelman, H. M., 1988. Comparison of several methods for calculating vibration mode shape derivatives. AIAA Journal 26 (12), 1506-1511.

Wang, B. P., 1991. Improved approximate methods for computing eigenvector derivatives in structural dynamics. AIAA Journal 29 (6), 1018-1020.

Wu, Z., Weaver, P. M., Raju, G., Chul Kim, B., 2012. Buckling analysis and optimisation of variable angle tow composite plates. Thin-Walled Structures $60,163-172$.

Zhou, X.-Y., Gosling, P. D., 2018. Influence of stochastic variations in manufacturing defects on the mechanical performance of textile composites. Composite Structures 194, 226-239.

Zhou, X. Y., Gosling, P. D., Ullah, Z., Kaczmarczyk, L., Pearce, C. J., 2017. Stochastic multi-scale finite element based reliability analysis for laminated composite structures. Applied Mathematical Modelling 45, 457-473. 


\section{List of Tables}

$551 \quad 1$ Comparisons of buckling eigenvalues obtained from in-house program and ANSYS . . . . . . . . . . . . . . 34

553

2 Mean values (MV) and standard deviations (STD) of buckling eigenvalues and critical buckling coefficients for VAT plate due to variations in material properties . . . . . . . . . . 34

3 Mean values and standard deviations of buckling eigenvalues and critical buckling coefficients for VAT plate due to variations in fibre tow path ............. . . 35 
Table 1: Comparisons of buckling eigenvalues obtained from in-house program and ANSYS

\begin{tabular}{|c|c|c|c|c|c|}
\hline \multirow{3}{*}{ Layup } & \multirow{2}{*}{ Mode } & \multicolumn{2}{|c|}{ Case I } & \multicolumn{2}{c|}{ Case II } \\
\cline { 3 - 6 } & & ANSYS & Inhouse & ANSYS & Inhouse \\
\hline \multirow{5}{*}{ Single } & 1 & $2.7181 \mathrm{e}-7$ & $2.7148 \mathrm{e}-7$ & $8.4566 \mathrm{e}-7$ & $8.4864 \mathrm{e}-7$ \\
\cline { 2 - 6 } & 2 & $5.5561 \mathrm{e}-7$ & $5.4834 \mathrm{e}-7$ & $8.5250 \mathrm{e}-7$ & $8.5304 \mathrm{e}-7$ \\
\cline { 2 - 6 } & 3 & $6.8151 \mathrm{e}-7$ & $6.7786 \mathrm{e}-7$ & $1.1386 \mathrm{e}-6$ & $1.1384 \mathrm{e}-6$ \\
\cline { 2 - 6 } & 4 & $7.8559 \mathrm{e}-7$ & $7.8298 \mathrm{e}-7$ & $1.4290 \mathrm{e}-6$ & $1.4267 \mathrm{e}-6$ \\
\hline \multirow{5}{*}{ 12-layer } & 5 & $1.1699 \mathrm{e}-6$ & $1.1493 \mathrm{e}-6$ & $1.8525 \mathrm{e}-6$ & $1.8588 \mathrm{e}-6$ \\
\hline & 1 & $2.2802 \mathrm{e}-5$ & $2.2798 \mathrm{e}-5$ & $1.3608 \mathrm{e}-4$ & $1.3579 \mathrm{e}-4$ \\
\cline { 2 - 6 } & 2 & $4.3446 \mathrm{e}-5$ & $4.3443 \mathrm{e}-5$ & $1.3708 \mathrm{e}-4$ & $1.3689 \mathrm{e}-4$ \\
\hline & 3 & $5.5295 \mathrm{e}-5$ & $5.5239 \mathrm{e}-5$ & $1.8683 \mathrm{e}-4$ & $1.8669 \mathrm{e}-4$ \\
\hline & 4 & $7.0165 \mathrm{e}-5$ & $7.0110 \mathrm{e}-5$ & $2.6192 \mathrm{e}-4$ & $2.6180 \mathrm{e}-4$ \\
\hline & 5 & $8.5142 \mathrm{e}-5$ & $8.5163 \mathrm{e}-5$ & $2.7680 \mathrm{e}-4$ & $2.7565 \mathrm{e}-4$ \\
\hline
\end{tabular}

Table 2: Mean values (MV) and standard deviations (STD) of buckling eigenvalues and critical buckling coefficients for VAT plate due to variations in material properties

\begin{tabular}{|c|c|c|c|c|c|}
\hline \multirow{2}{*}{ Case } & \multirow{2}{*}{ Method } & \multicolumn{2}{|c|}{$\lambda$} & \multicolumn{2}{c|}{$K_{c r}$} \\
\cline { 3 - 6 } & & MV & STD & MV & STD \\
\hline \multirow{2}{*}{ I } & MCS & $2.2295 \mathrm{e}-5$ & $8.7518 \mathrm{e}-8$ & 1.3834 & 0.1265 \\
\cline { 3 - 6 } & PSFE & $2.2294 \mathrm{e}-5$ & $8.6747 \mathrm{e}-8$ & 1.3824 & 0.1252 \\
\hline \multirow{2}{*}{ II } & MCS & $1.3502 \mathrm{e}-4$ & $1.1473 \mathrm{e}-5$ & 3.0273 & 0.3594 \\
\cline { 2 - 6 } & PSFE & $1.3479 \mathrm{e}-4$ & $1.1426 \mathrm{e}-5$ & 3.0315 & 0.3533 \\
\hline
\end{tabular}


Table 3: Mean values and standard deviations of buckling eigenvalues and critical buckling coefficients for VAT plate due to variations in fibre tow path

\begin{tabular}{|c|c|c|c|c|c|}
\hline \multirow{2}{*}{ Case } & \multirow{2}{*}{ Method } & \multicolumn{2}{|c|}{$\lambda$} & \multicolumn{2}{c|}{$K_{c r}$} \\
\cline { 3 - 6 } & & MV & STD & MV & STD \\
\hline \multirow{3}{*}{ I } & MCS & $2.2208 \mathrm{e}-5$ & $5.0548 \mathrm{e}-8$ & 1.3771 & 0.0055 \\
\cline { 2 - 6 } & PSFE & $2.2207 \mathrm{e}-5$ & $5.6706 \mathrm{e}-8$ & 1.3769 & 0.0057 \\
\hline \multirow{3}{*}{ II } & MCS & $1.3238 \mathrm{e}-4$ & $4.5574 \mathrm{e}-6$ & 2.9988 & 0.0346 \\
\cline { 2 - 6 } & PSFE & $1.3258 \mathrm{e}-4$ & $5.0503 \mathrm{e}-6$ & 2.9979 & 0.0382 \\
\hline
\end{tabular}




\section{List of Figures}

1 Configuration of the variable angle tow composite laminate. . 38

2 Discretised fibre path with linear variation formula: $0^{\circ}<$

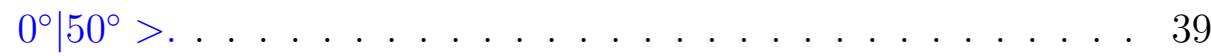

3 In-plane deformation profile of Case I and Case II panels under uniform end shortening. . . . . . . . . . . . . . . 39

4 Coefficients of variation of normalized buckling factors from MCS and PSFE with uncertainties in material properties with normal distributions . . . . . . . . . . . . . . . . 40

5 Coefficients of variation of normalized buckling factors from MCS and PSFE with uncertainties in fibre tow path with uniform distributions . . . . . . . . . . . . . . . . . 40

6 Buckling statistics of Case I panel under variations in material

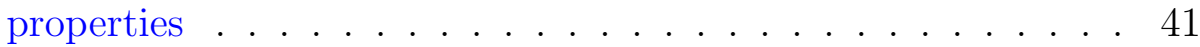

7 Buckling statistics of Case II panel under variations in material properties ..................... . . . 42

8 The first two buckling mode shapes under different $T_{1}$ : (a) and (b) for $T_{1}=73^{\circ}$; (c) and (d) for $T_{1}=74^{\circ} \ldots \ldots . . . . .43$

9 The first order partial derivative of the first two lowest $\lambda$ and $K_{c r}$ with respect to $E_{1} \ldots \ldots \ldots \ldots 4$

10 Buckling statistics of Case I panel under variations in fibre

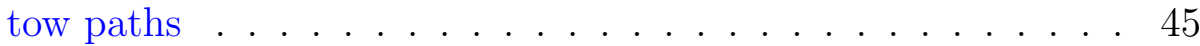

11 The first order partial derivatives of $\lambda$ and $K_{c r}$ with respect to fibre tow path parameters for Case I panel . . . . . . . . . 46 
12 Buckling statistics of Case II panel under variations in fibre

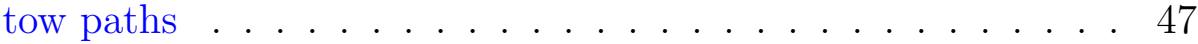

13 The first order partial derivatives of $\lambda$ and $K_{c r}$ with respect to fibre tow path parameters for Case II panel . . . . . . . . . 48

14 Discontinuous region of the first order partial derivatives of $\lambda$

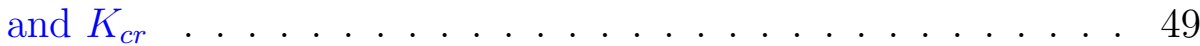

15 Sensitivity factors of material properties for buckling statistics. 50

16 Sensitivity factors of fibre tow path parameters for buckling statistics. .................... 50 


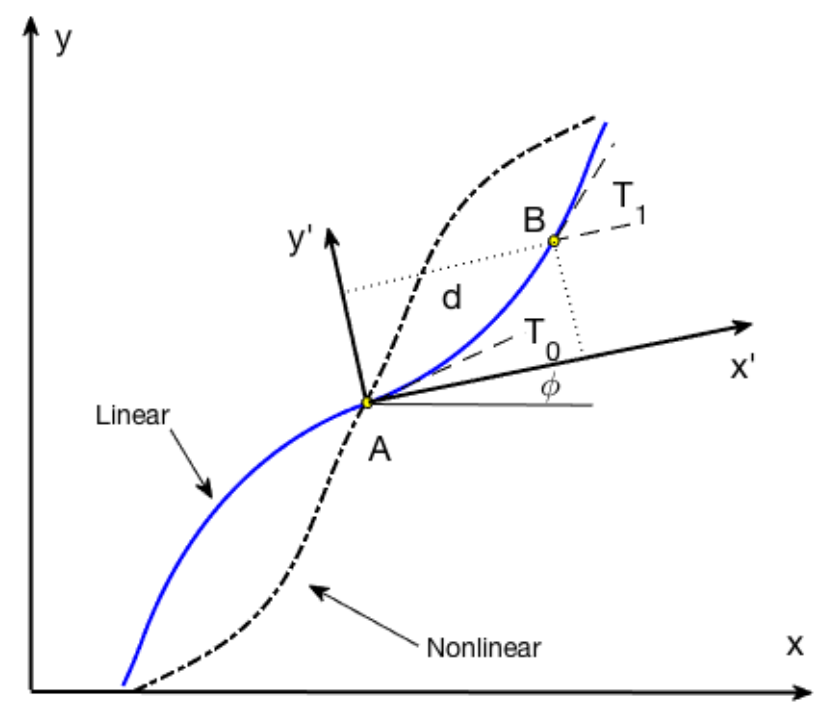

(a) Fibre path functions

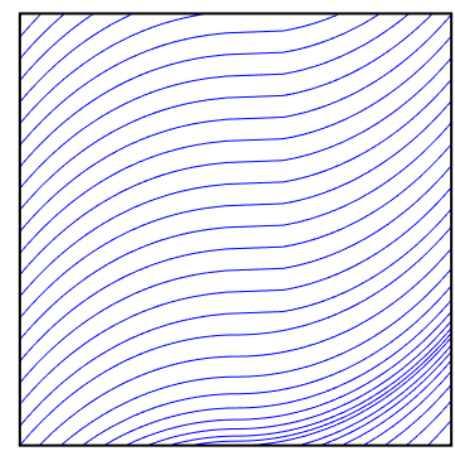

(b) Linear variation

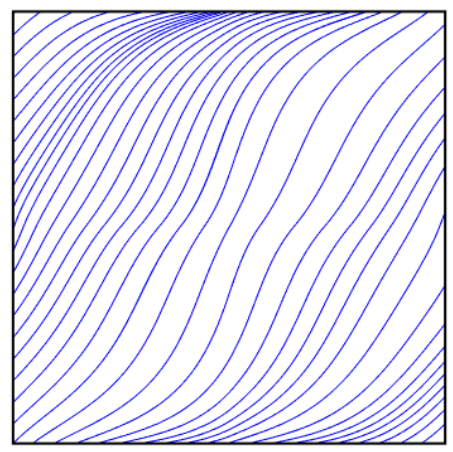

(c) Nonlinear variation

Figure 1: Configuration of the variable angle tow composite laminate. 


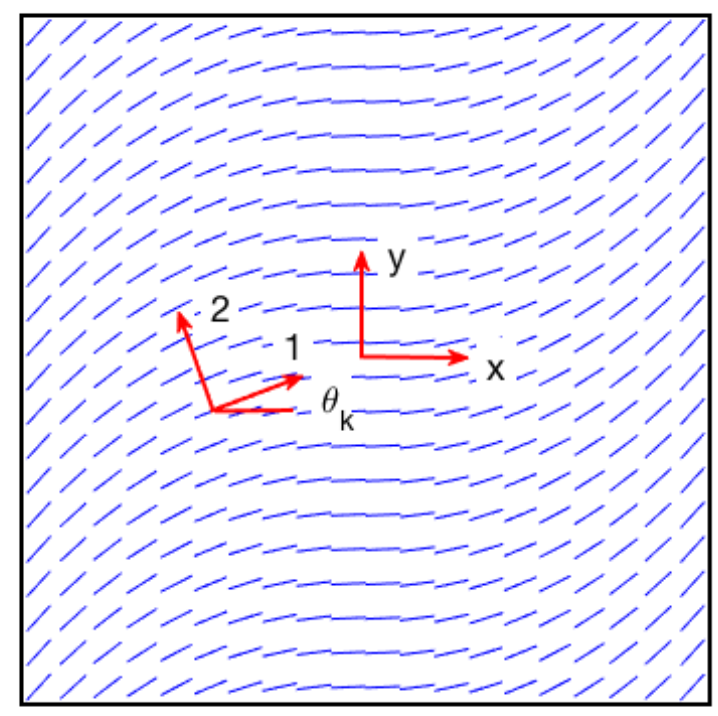

Figure 2: Discretised fibre path with linear variation formula: $0^{\circ}<0^{\circ} \mid 50^{\circ}>$.

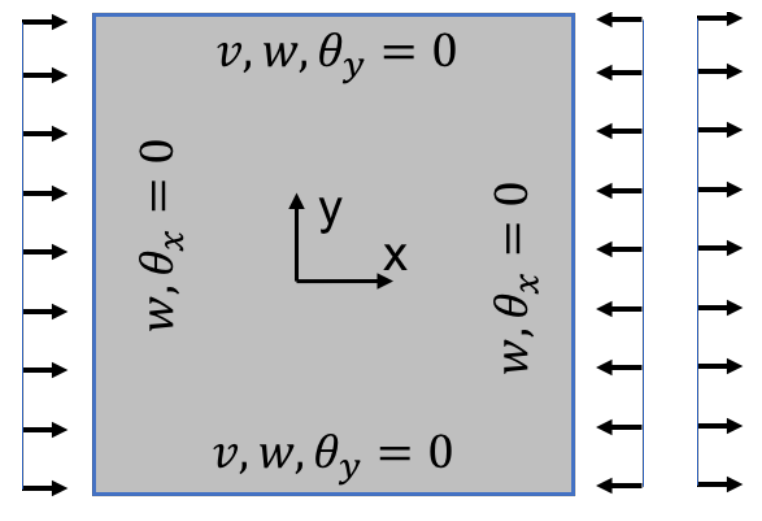

(a) Case I panel

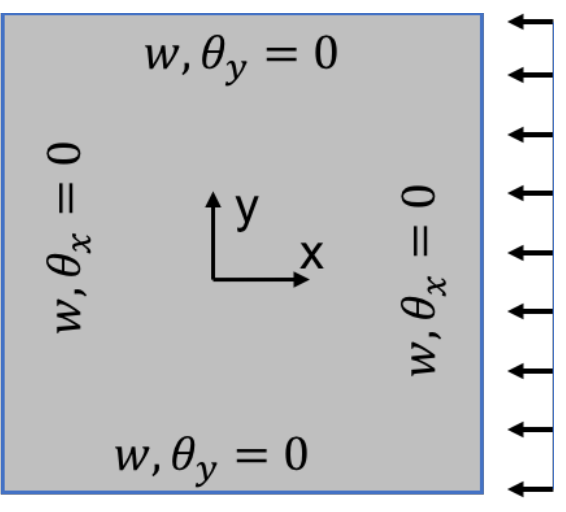

(b) Case II panel

Figure 3: In-plane deformation profile of Case I and Case II panels under uniform end shortening. 


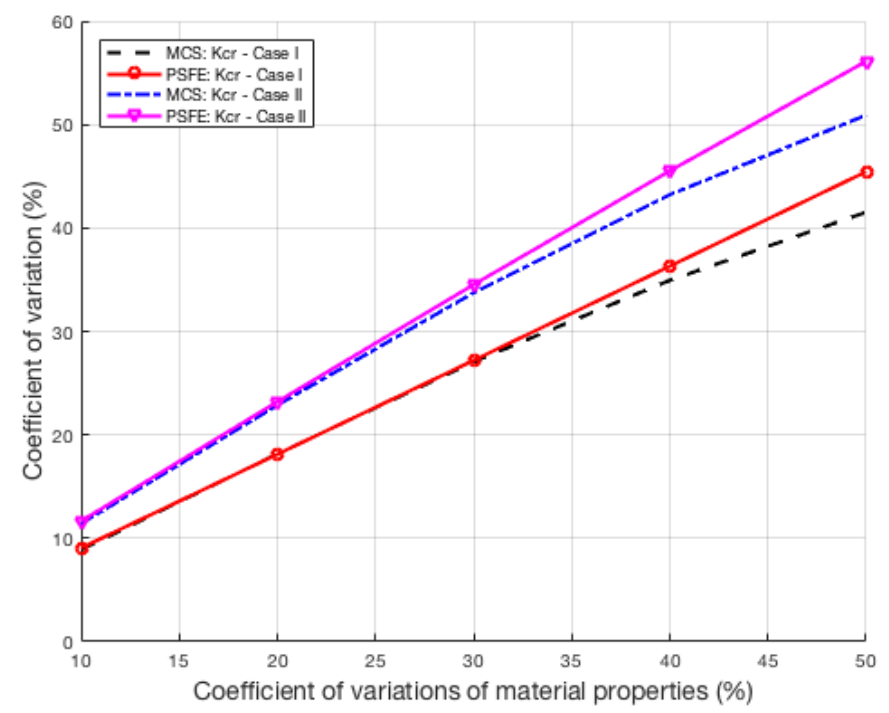

Figure 4: Coefficients of variation of normalized buckling factors from MCS and PSFE with uncertainties in material properties with normal distributions

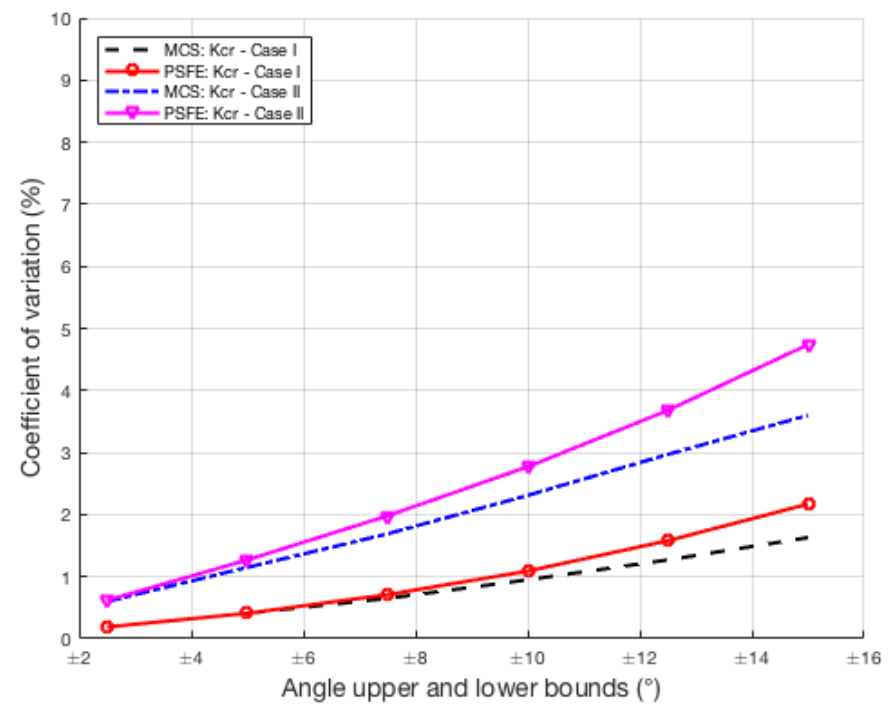

Figure 5: Coefficients of variation of normalized buckling factors from MCS and PSFE with uncertainties in fibre tow path with uniform distributions 

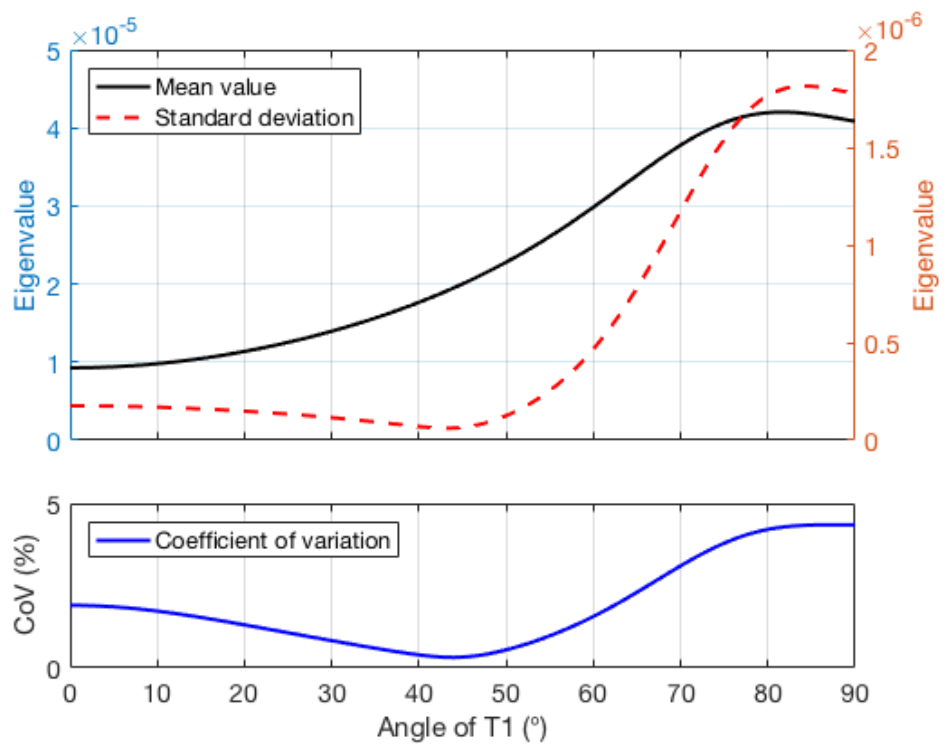

(a) Eigenvalues
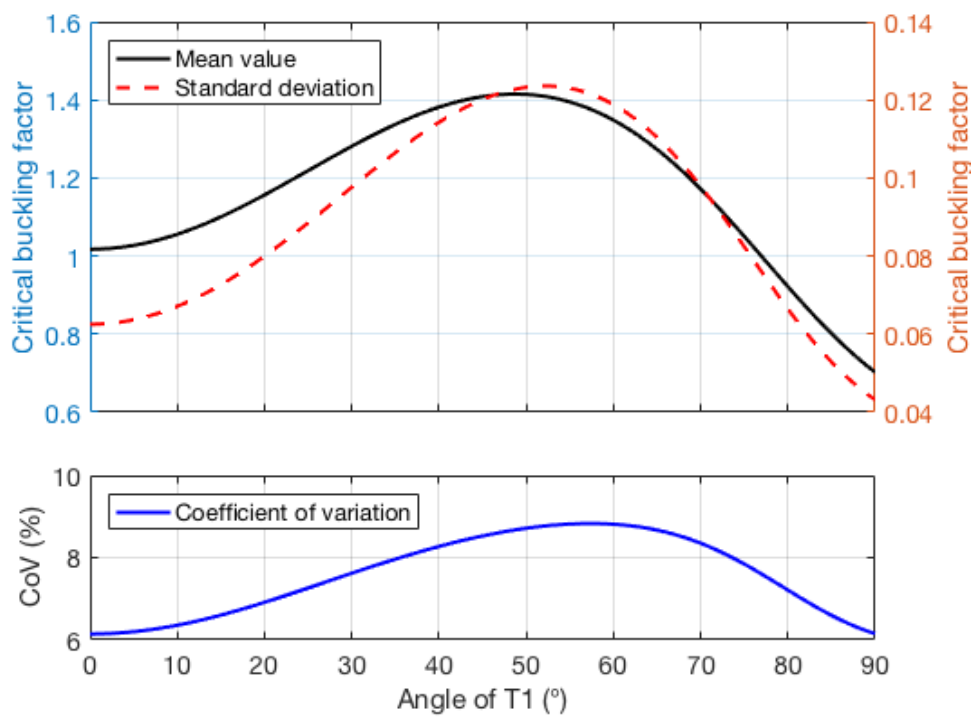

(b) Critical buckling coefficients

Figure 6: Buckling statistics of Case I panel under variations in material properties 

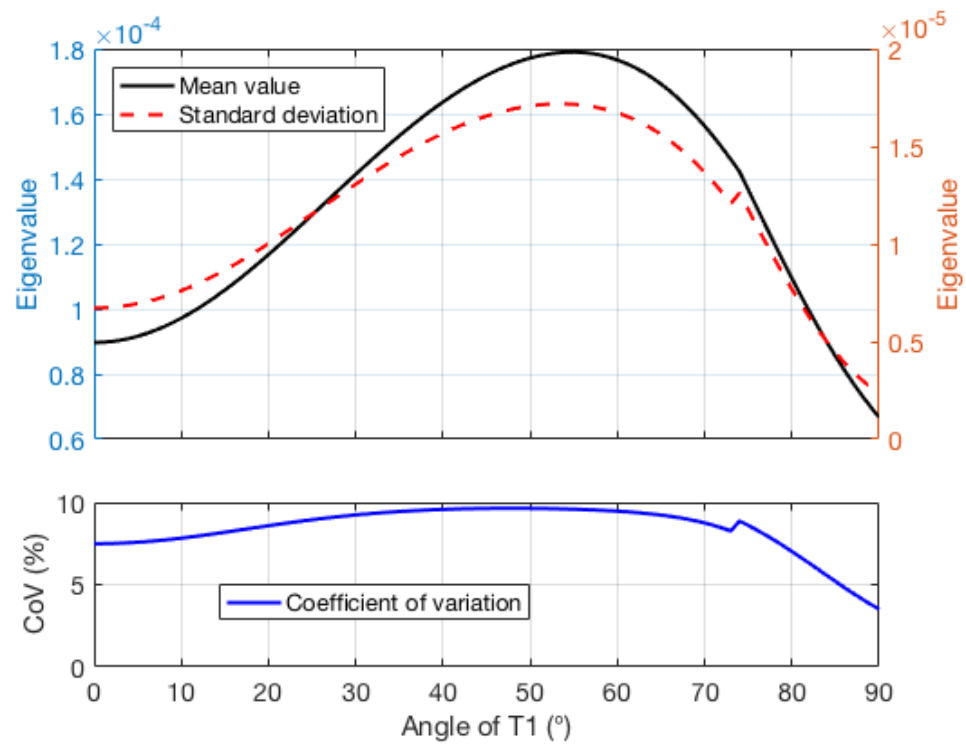

(a) Eigenvalues
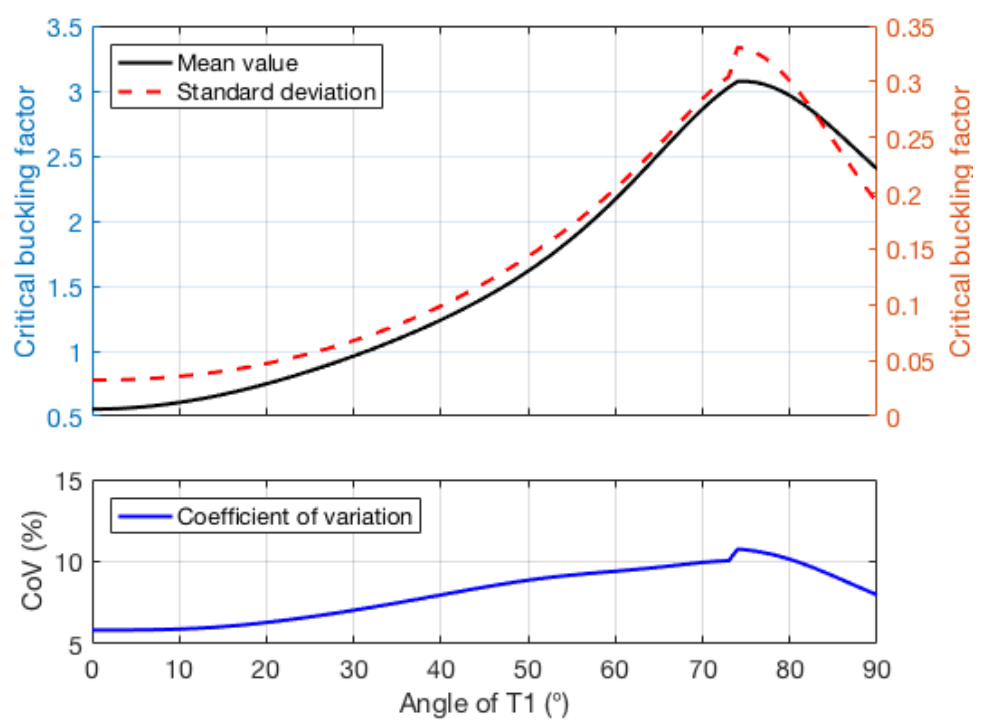

(b) Critical buckling coefficients

Figure 7: Buckling statistics of Case II panel under variations in material properties 


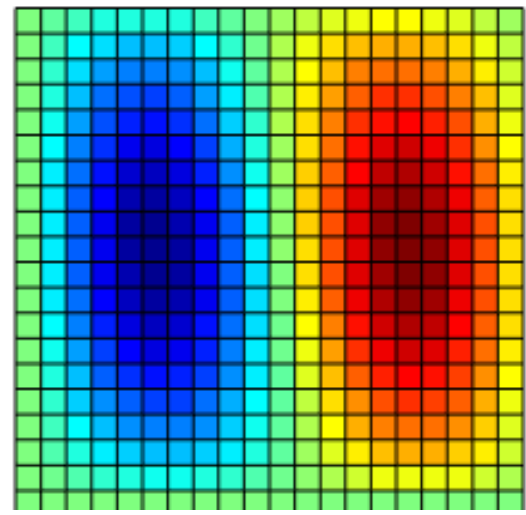

(a) 1st mode shape, $K_{c r}=3.0240$

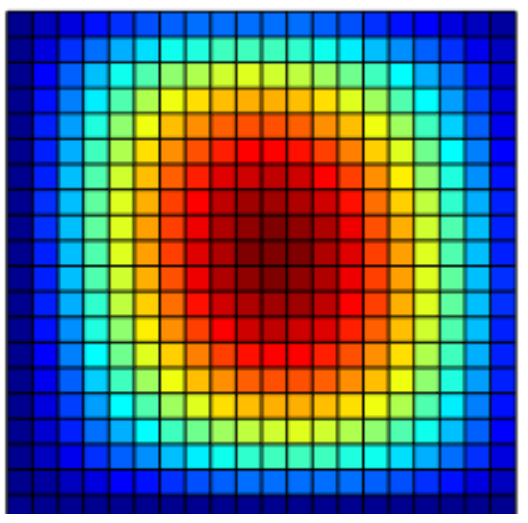

(c) 1st mode shape, $K_{c r}=3.0673$

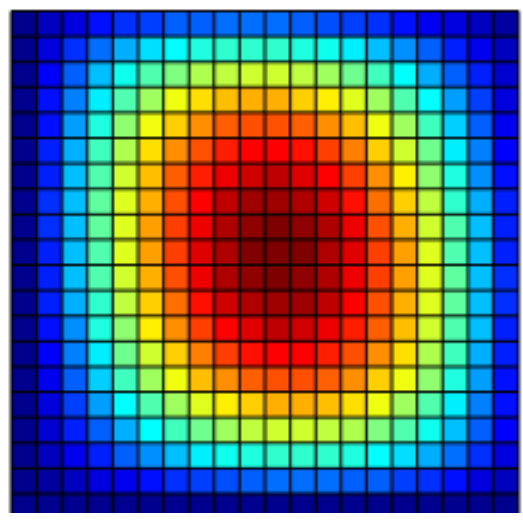

(b) 2nd mode shape, $K_{c r}=3.02594$

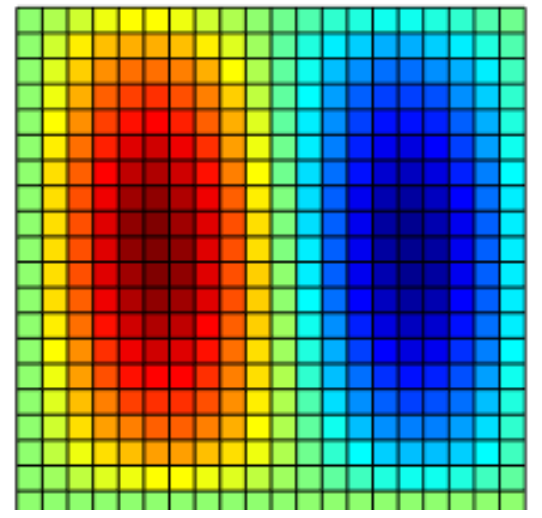

(d) 2nd mode shape, $K_{c r}=3.0679$

Figure 8: The first two buckling mode shapes under different $T_{1}$ : (a) and (b) for $T_{1}=73^{\circ}$; (c) and (d) for $T_{1}=74^{\circ}$. 


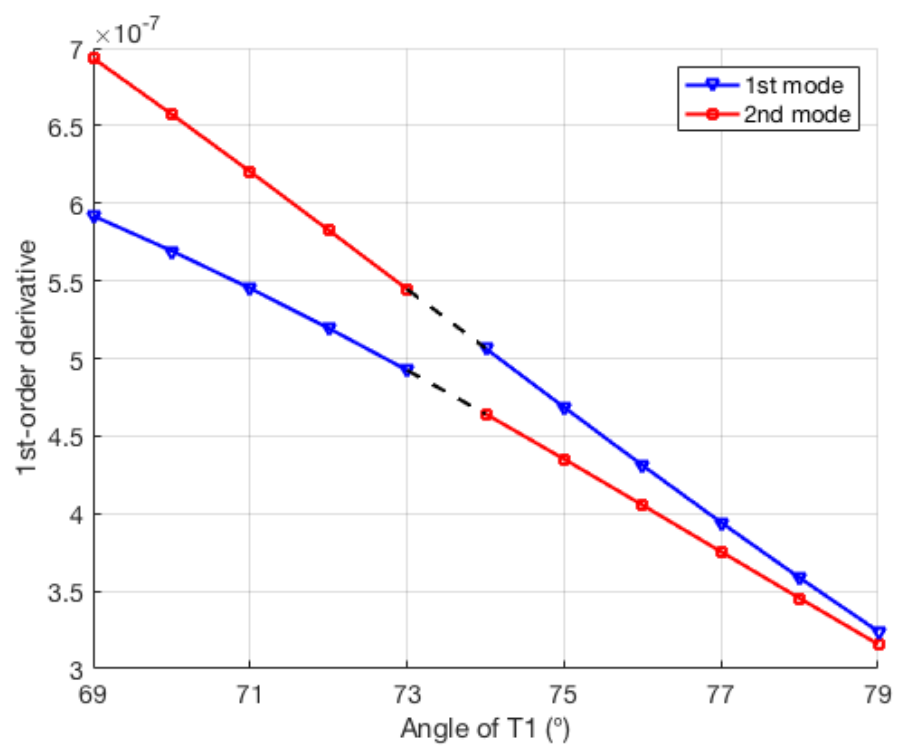

(a) Eigenvalues

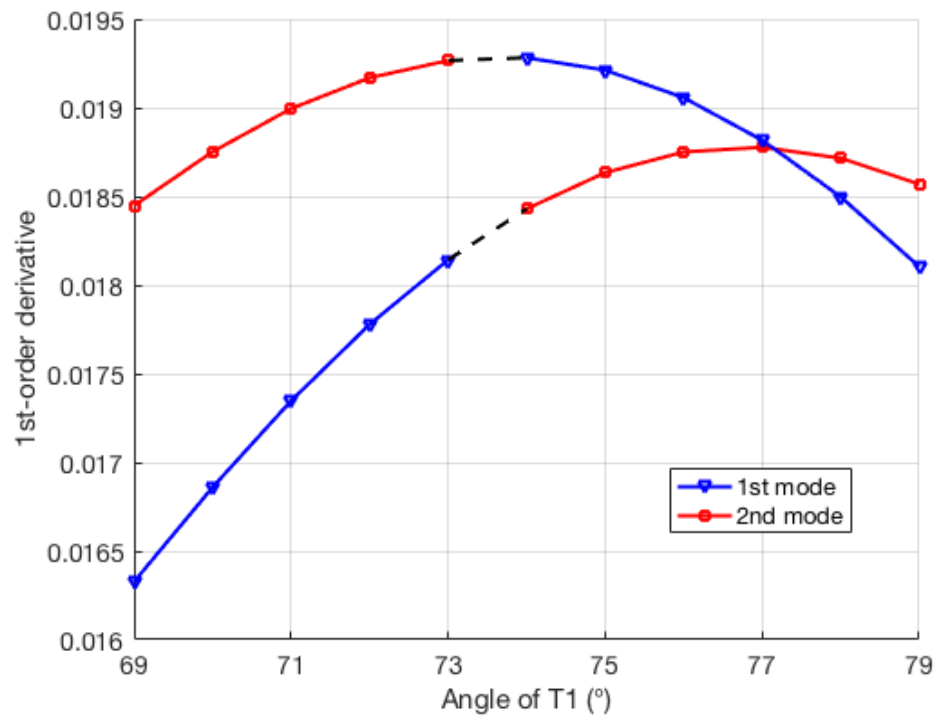

(b) Critical buckling coefficients

Figure 9: The first order partial derivative of the first two lowest $\lambda$ and $K_{c r}$ with respect to $E_{1}$. 

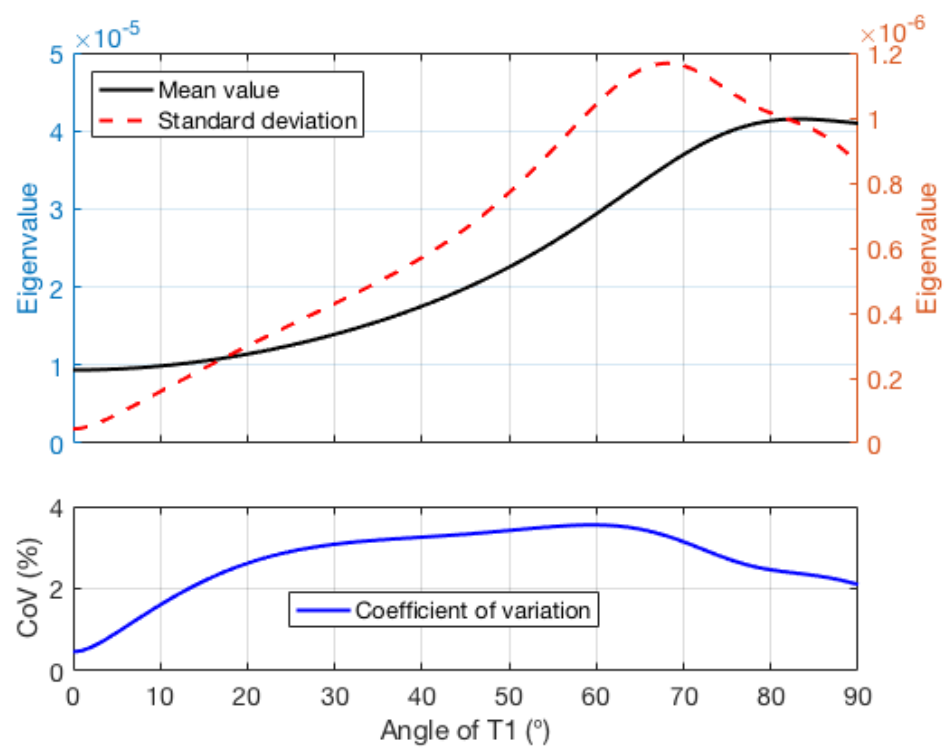

(a) Eigenvalues
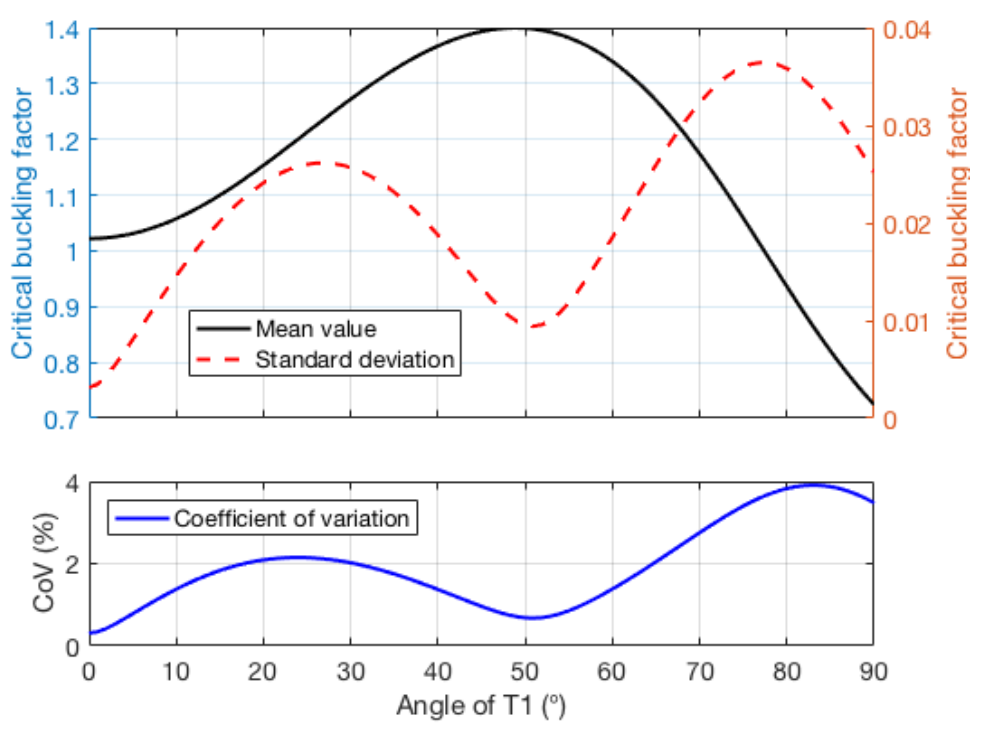

(b) Critical buckling coefficients

Figure 10: Buckling statistics of Case I panel under variations in fibre tow paths 


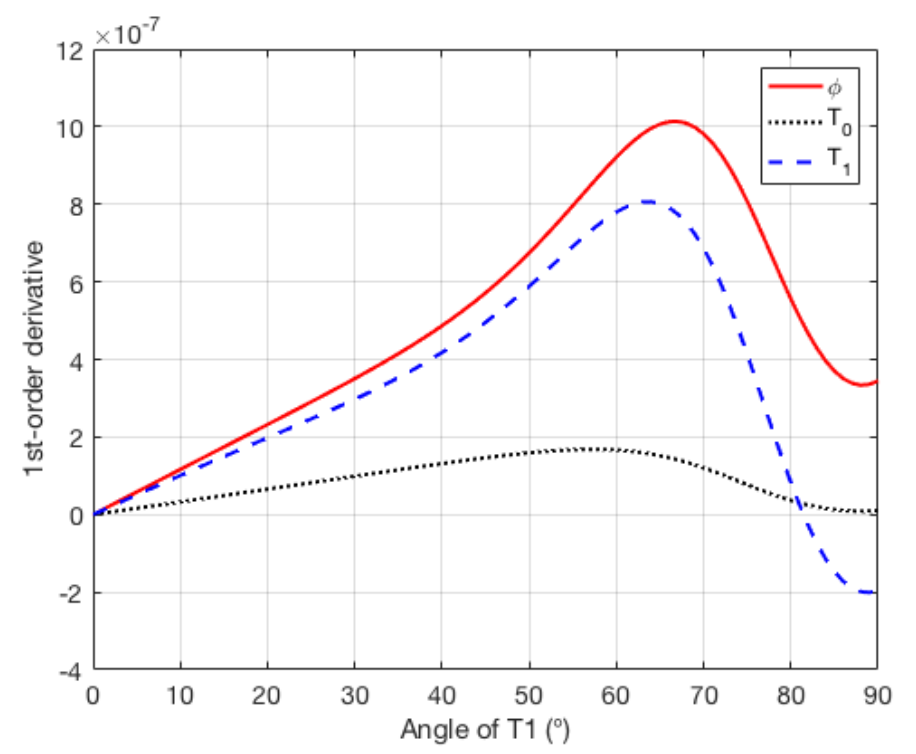

(a) Eigenvalues

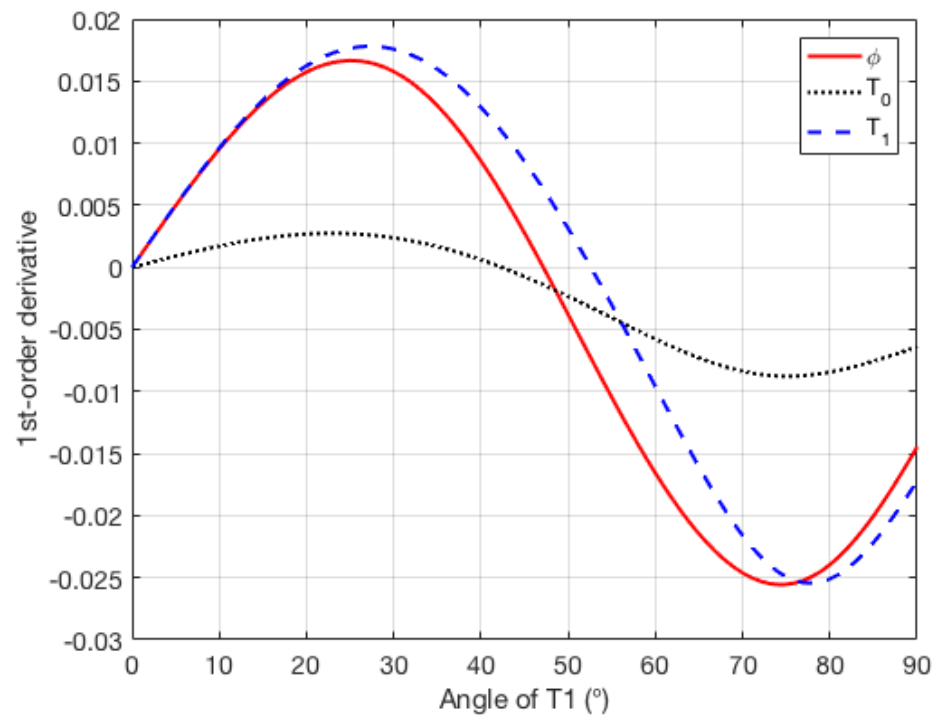

(b) Critical buckling coefficients

Figure 11: The first order partial derivatives of $\lambda$ and $K_{c r}$ with respect to fibre tow path parameters for Case I panel 

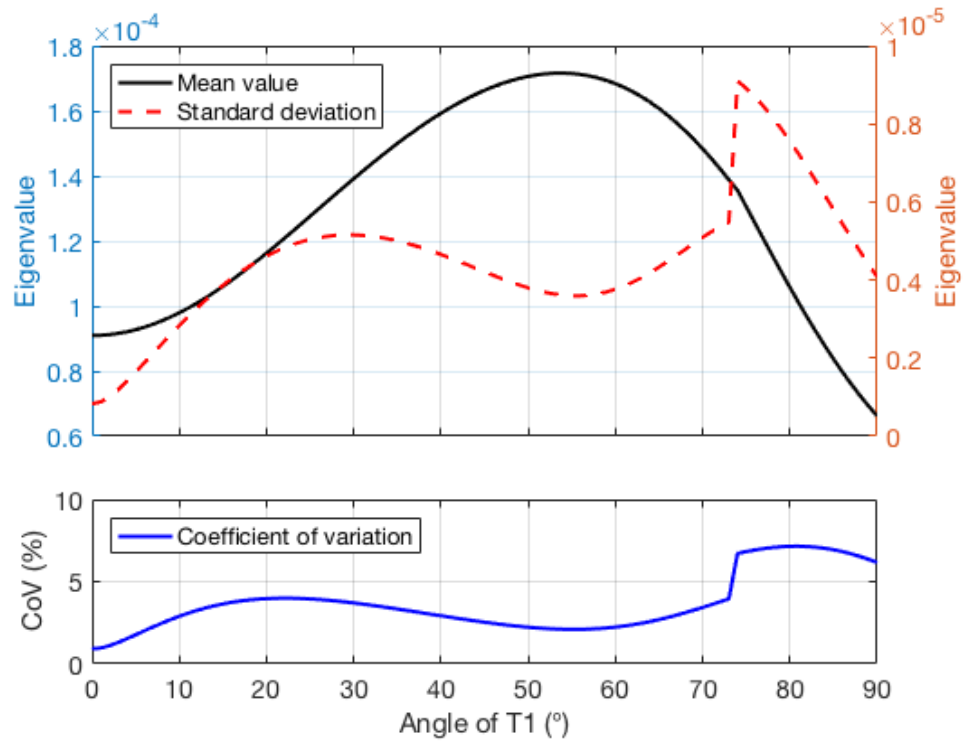

(a) Eigenvalues
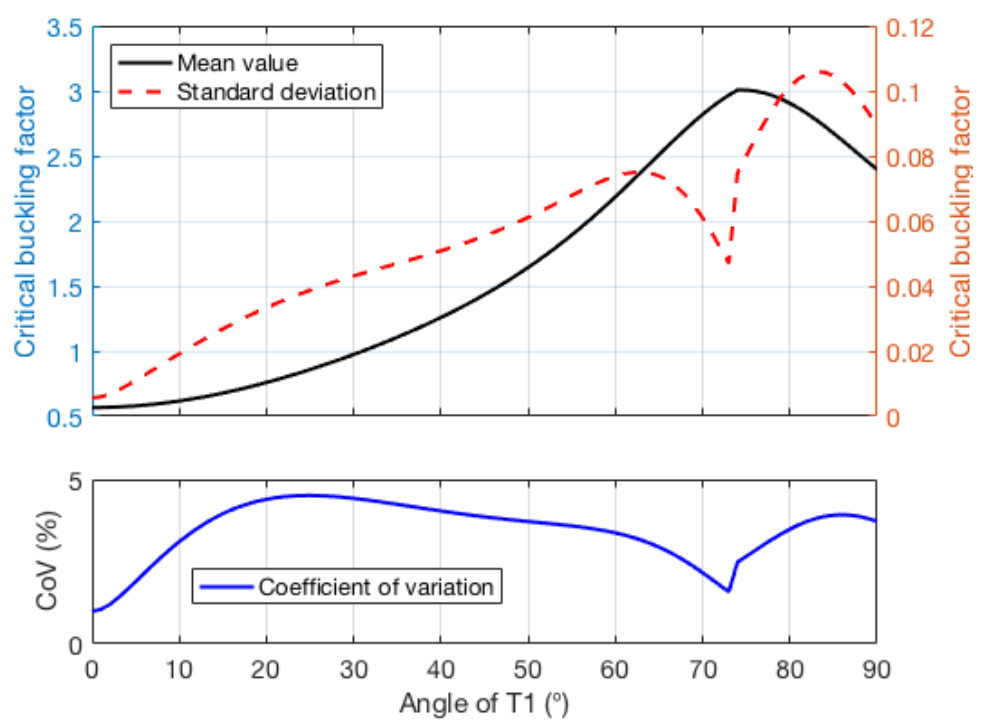

(b) Critical buckling coefficients

Figure 12: Buckling statistics of Case II panel under variations in fibre tow paths 


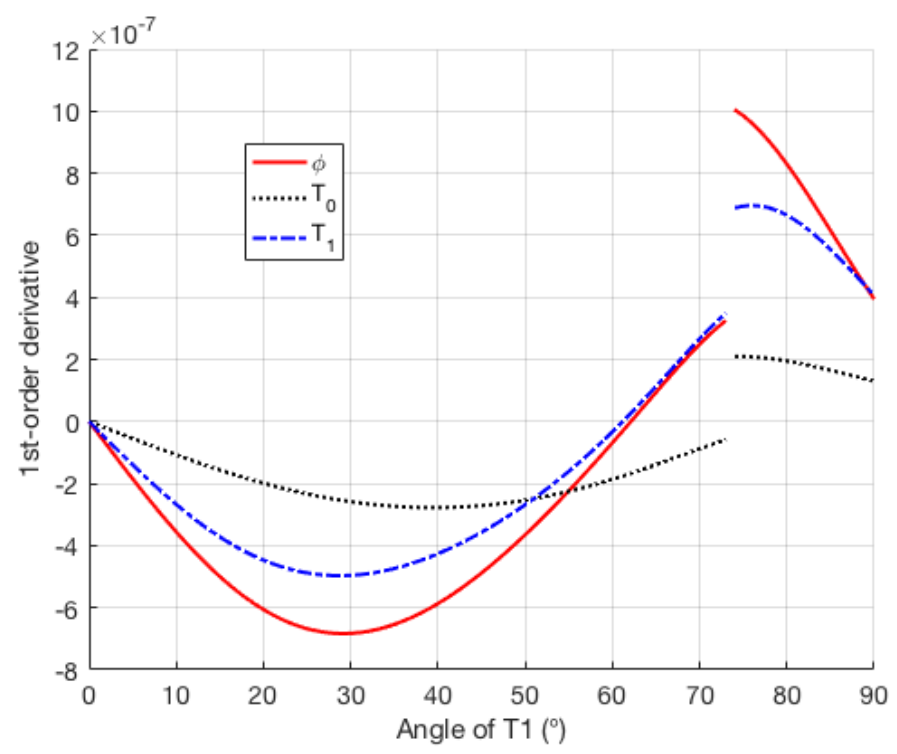

(a) Eigenvalues

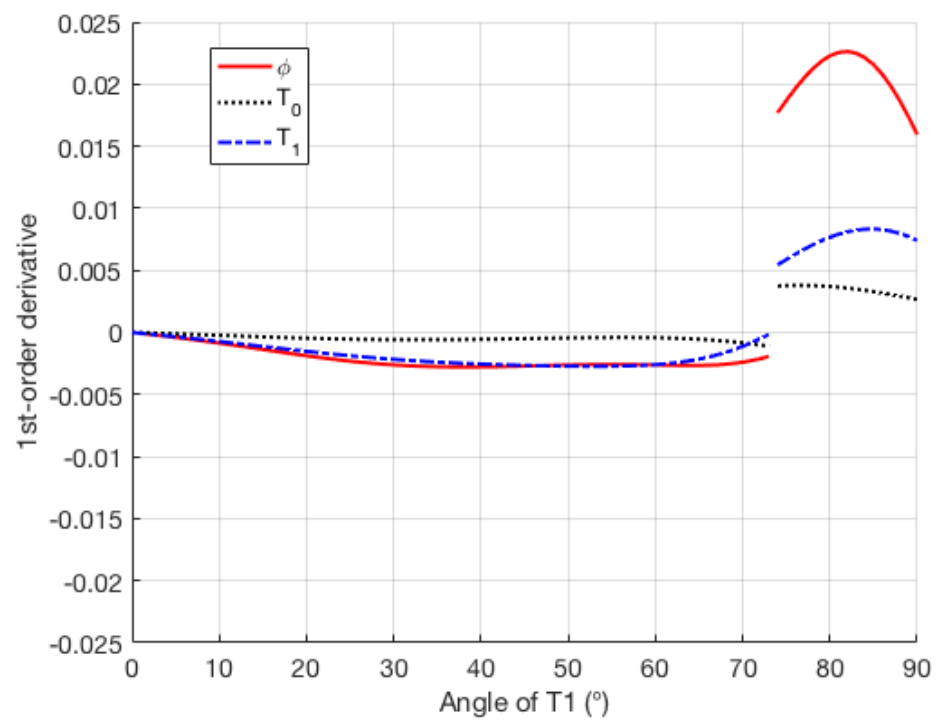

(b) Critical buckling coefficients

Figure 13: The first order partial derivatives of $\lambda$ and $K_{c r}$ with respect to fibre tow path parameters for Case II panel 


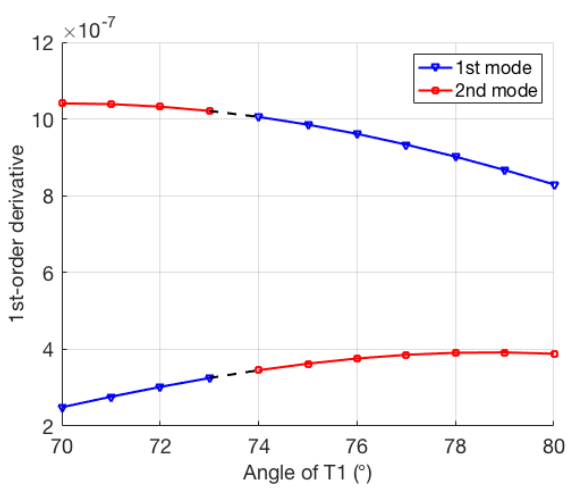

(a) $\partial \lambda / \partial p h i$

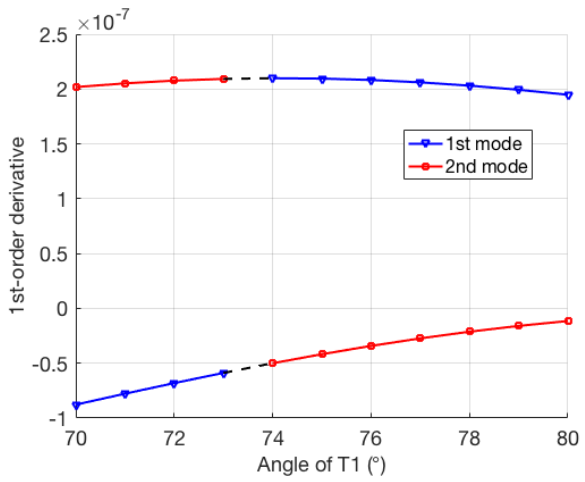

(c) $\partial \lambda / \partial T_{0}$

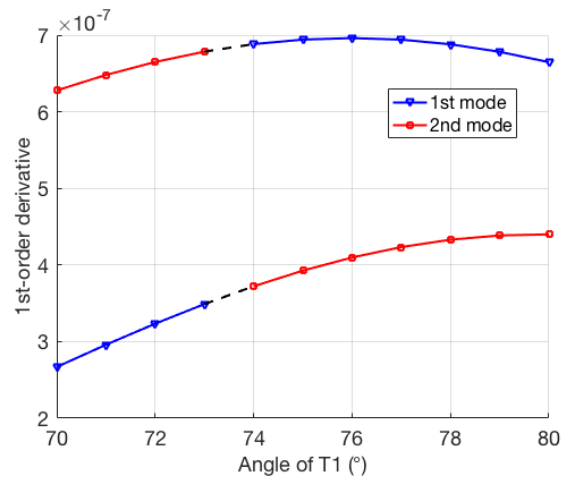

(e) $\partial \lambda / \partial T_{1}$

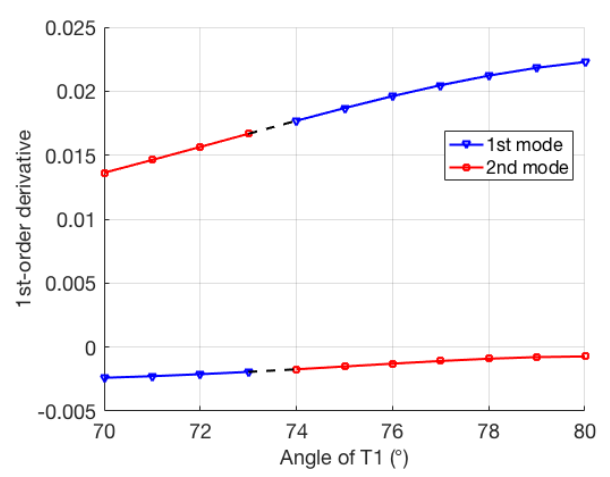

(b) $\partial K_{c r} / \partial p h i$

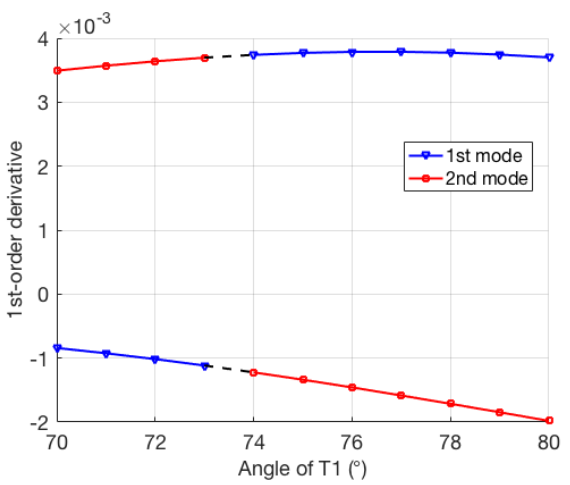

(d) $\partial K_{c r} / \partial T_{0}$

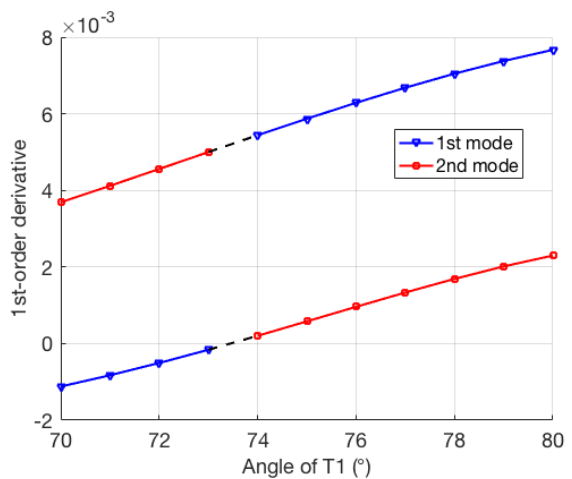

(f) $\partial K_{c r} / \partial T_{1}$

Figure 14: Discontinuous region of the first order partial derivatives of $\lambda$ and $K_{c r}$ 


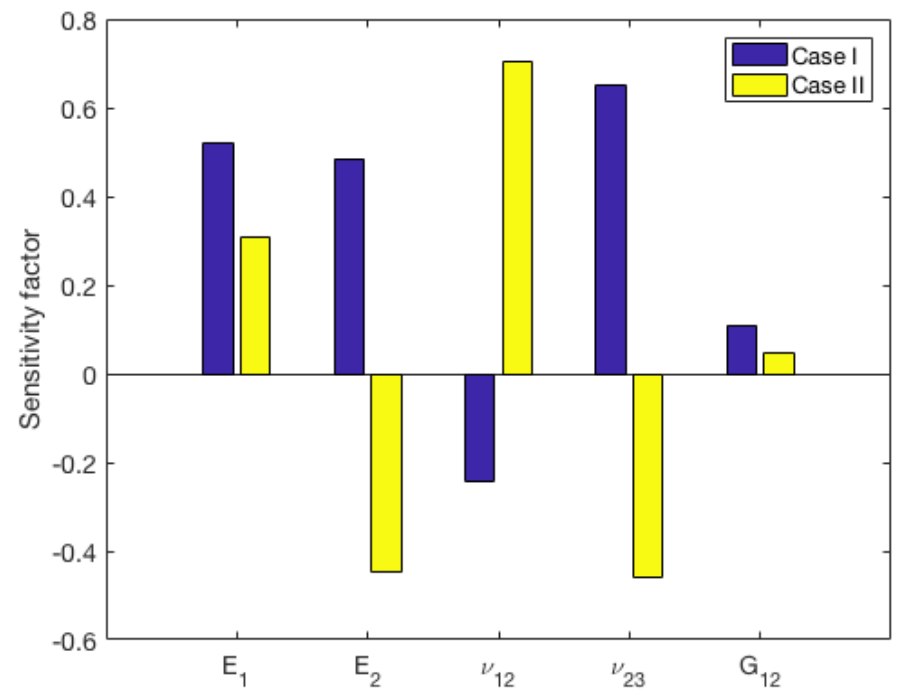

Figure 15: Sensitivity factors of material properties for buckling statistics.

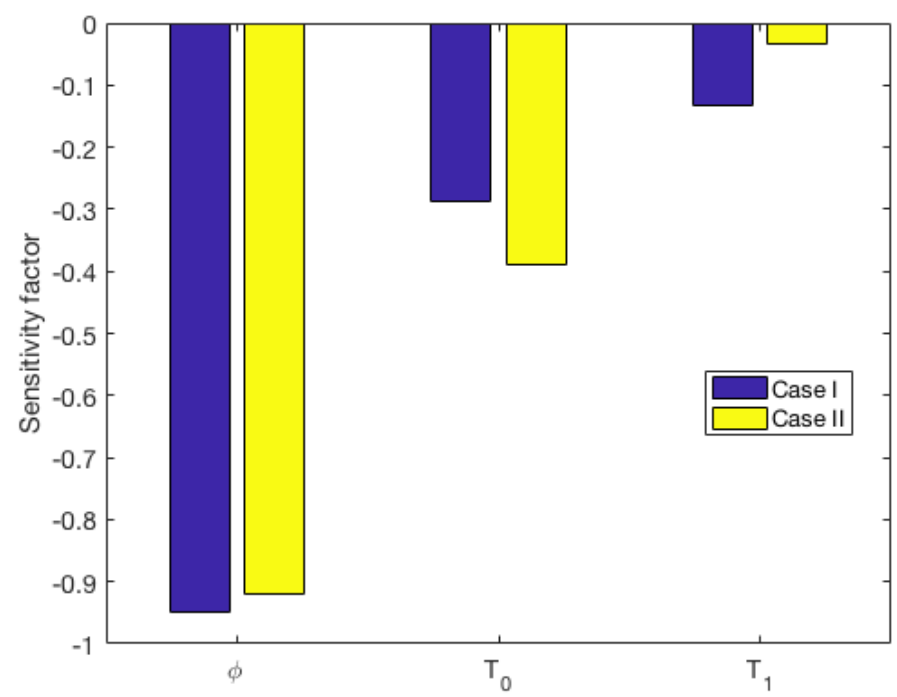

Figure 16: Sensitivity factors of fibre tow path parameters for buckling statistics. 